\title{
PHYSIOLOGICAL RESPONSE OF MAIZE HYBRIDS TO NITROGEN AND
} PHOSPHORUS FERTILIZATION

\author{
Hytham M. El-Shahed $^{1 *}$, S. A. Mowafy ${ }^{2}$, M.M.A. Osman ${ }^{1}$ and Nehal Z. El-Naggar ${ }^{2}$ \\ 1. Maize Res. Prog., Field Crops Res. Inst., ARC, Egypt \\ 2. Agron. Dept., Fac. Agric., Zagazig Univ., Egypt
}

Received: 01/11/2016 ; Accepted: 11/01/2017

\begin{abstract}
Two field experiments were conducted at the Experimental Farm, Gemmeiza Agriculture Research station, Agricultural Research Center white maize (ARC), Egypt during two growing seasons (2014 and 2015), to study the response of two maize hybrids i.e., white maize single cross 128 and yellow maize single cross 176 to four nitrogen fertilizer levels i.e. $0,45,90$ and $135 \mathrm{~kg}$ $\mathrm{N} /$ fad., and three phosphorus fertilizer levels i.e. $0,15.5$ and $31 \mathrm{~kg} \mathrm{P}_{2} \mathrm{O}_{5} /$ fad. Concerning the obtained results, SC 128 outyielded SC 176 in grain and biological yields (combined data) due to superiority in days to $50 \%$ tassiling and silking, leaf chlorophyll content, ear leaf area, leaf area index, relative photosynthetic potential for stover yield/plant, ear length and diameter, stem diameter, number of grains/row, hundred grain weight as well as grain and stover yields/plant, while SC. 176 gave the highest mean for each of plant height and relative photosynthetic potential for grain yield/plant. No significant differences among maize hybrid could be detected in relative photosynthetic potential for biological yield/plant, number of rows/ear, ear leaf efficiency and migration coefficient. Data of combined analysis revealed that addition of nitrogen $(\mathrm{N})$ fertilizer and raising its rate up to $135 \mathrm{~kg}$ $\mathrm{N} /$ fad., was accompanied by asignificant increase in each of leaf chlorophyll content $\left(\mathrm{mg} \mathrm{m}^{-2}\right)$, plant height, leaf area index (LAI), ear length and diameter, stem diameter, number of rows/ear, number of grains/row, hundred grain weight, grain and stover yields/plant, ear leaf efficacy and migration coefficient, but relative photosynthetic potential for both stover and biological yields/plant were decreased. Increase of phosphorus (P) level up to $31 \mathrm{~kg}_{2} \mathrm{O}_{5} /$ fad., was followed by a significant increase in grain yield/fad., and almost all their attributes, but relative photosynthetic potential for both stover and biological yields/plant were decreased. Meantime, ear diameter responded to phosphorus addition up to $15.5 \mathrm{~kg} \mathrm{P}_{2} \mathrm{O}_{5} /$ fad., however, days to $50 \%$ tassling and silking were not significantly affected by varying phosphorus levels according to combined analysis. In almost cases, maize hybrid SC. 128 was more efficient and more responsive than SC. 176, where the former was is need to $135 \mathrm{~kg}$ $\mathrm{N} /$ fad., and $31 \mathrm{~kg} \mathrm{P}_{2} \mathrm{O}_{5} /$ fad., in order to maximize the grain yield to only $42.8 \mathrm{ardab} / \mathrm{fad}$.
\end{abstract}

Key words: Zea mays L., hybrids, nitrogen fertilizer, phosphate fertilizer, agronomic characters; Egypt.

\section{INTRODUCTION}

Maize (Zea mays L.) is the world's widely grown highland cereal and primary staple food crop in many developing countries. It is the third most important staple food crop in both area and production after wheat and rice in Egypt. Total area under cultivation of maize in Egypt is 857329 hectare which is about $25.00 \%$ of the total cultivated agricultural land while average yield is 8.40 ton $\mathrm{ha}^{-1}$. (FAOSTAT, 2015). The rapidly increasing demand of maize is driven by the increased demand for direct human consumption in the hills as a staple food crop (Ghimire et al., 2007). The Egyptian government aims to decrease the gap between consumption and production via increasing grain yield per unit area. There are several approaches

* Corresponding author: Tel. : + 201008318602

E-mail address: haythammostfa@yahoo.com 
to increase crop productivity, improving farming practices, employing merging technology, using new and high yielding maize hybrids which have more efficiently for using nitrogen and more response to high rate of nitrogenous fertilizer to create more grain.

The hybrid maize gave higher yields and using $\mathrm{N}$ and $\mathrm{P}$ more efficiently than the open pollinated one at both trial locations and hybrid varieties responding up to $200 \mathrm{~kg} \mathrm{~N}^{-1}$ (Kogbe and Adediran, 2003). Also, (El-Sheikh, 1998) reported that application of $160 \mathrm{~kg} \mathrm{~N} \mathrm{ha}{ }^{-1}$ significantly increased grain yield of maize. On the contrary, nitrogen deficiency decreased grain yield for all hybrids, especially the older hybrids. However, there were no significant differences regarding harvest index, leaf area or plant weight at flowering stage between the Ndeficient and control plants of all hybrids. Dry matter production after flowering of the nitrogen deficient plants was, significantly, lower than that of the control plants in all hybrids, also, $\mathrm{N}$ deficiency accelerated senescence, i.e. decreased chlorophyll and protein contents, after anthesis more for the earlier released hybrids than for the later ones (Ding et al., 2005).

However, nitrogenous fertilizere is one of the most important factors for crop growth, high yield, yield components and grain quality. Where, nitrogen element plays an essential role in many compounds essential for plant growth, chlorophyll and many enzymes. It is considered the key element in increasing crops productivity, also, helps in the use of $\mathrm{P}, \mathrm{K}$ and other elements in plants.

Additionally, (Bader and Othman, 2006) find out that increasing level of nitrogenous fertilization led to increase grain yield and its components. Increasing nitrogen fertilizer rate from zero up to $250 \mathrm{~kg} \mathrm{~N} \mathrm{ha}^{-1}$, significantly increased the studied maize growth, yield and yield components characters where maximum number of leaves/plant, number of cobs/plant, number of grains/cob, taller plants, grain and biological yields were recorded in ridge planting and application of $200 \mathrm{~kg} \mathrm{~N} \mathrm{ha}^{-1}$ when compared with other treatments (Bakht et al., 2006). Considering soil fertility status and cropping system, the $150 \mathrm{~kg} \mathrm{~N} \mathrm{ha}^{-1}$ application to maize variety Jalal in Peshawar was required for maximum biological and seed production (Akmal et al., 2010). Ears $\mathrm{m}^{-2}$, grains/ear, 1000 grain weight, grain yield, biological yield and harvest index were higher at the highest level of nitrogen (Arif et al., 2010).

Phosphorus plays a key role in energy transfer and is essential for photosynthesis and other chemo-physiological processes in plants. The different NP combinations significantly affected plant height, cob bearing plants, number of grains/cob, 1000-grain weight and grain yield, therefore the proper management of these two nutrient elements is very important for good crop production.

In this connection several research workers get significant response to phosphorus fertilization up to $35 \mathrm{~kg} \mathrm{P}_{2} \mathrm{O}_{5}$ ha $^{-1}$ (Diab et al., 1990; El-Far, 1996; Hegazy et al., 1996). However, other got higher response when they added $71 \mathrm{~kg} \mathrm{P}_{2} \mathrm{O}_{5}$ ha $^{-}$ ${ }^{1}$ (Badawi and El-Moursy, 1997; Salem, 2000; Hussein, 2009; Amanullah and Khalil, 2010). Furthermore, (Masood et al., 2011) get more higher response when they added $100 \mathrm{~kg} \mathrm{P}_{2} \mathrm{O}_{5}$ $\mathrm{ha}^{-1}$. Yosefi et al. (2011) reported that application of $50 \mathrm{~kg} \mathrm{P} \mathrm{ha}^{-1}$ with $100 \mathrm{~g}$ biophosphate gave the highest yield in Iran.

In order to achieve maximum attainable yield from the existing corn varieties in a given environment, it is essential to improve crop fertilizer management practices. The present study objective to determine a suitable combination of nitrogen and phosphorus fertilizers for maximizing grain yield of maize under Egyptian conditions.

\section{MATERIALS AND METHODS}

The current investigation was carried out at the Experimental Farm, Gemmeiza Agriculture Research Station, Gharbiya Governorate, Agricultural Research Center (ARC), Egypt during 2014 and 2015 summer growing seasons.

\section{The Studied Factors}

Factors under the study included two maize hybrids $(\mathrm{H})$ i.e. white single cross (S.C.) 128 and yellow single cross (SC) 176, four nitrogen (N) fertilizer levels which were 0, 45, 90 and $135 \mathrm{~kg} \mathrm{~N} /$ fad., as well as three phosphorus (P) levels i.e. $0,15.5$ and $31 \mathrm{~kg} \mathrm{P}_{2} \mathrm{O}_{5} /$ fad. 


\section{Cultural Practices}

Each plot consisted of five ridges, each ridge was $6 \mathrm{~m}$ long, $80 \mathrm{~cm}$ in width, $25 \mathrm{~cm}$ between hills and one blank ridge was left between plots. The outer two ridges ( $1^{\text {st }}$ and $5^{\text {th }}$ ) were considered as borders. The previous crop was wheat in both years. Planting was done on June $3^{\text {rd }}$ in 2014 season, and June $9^{\text {th }}$ in 2015 season. Ordinary super phosphate $\left(15.5 \% \quad \mathrm{P}_{2} \mathrm{O}_{5}\right)$, was applied before planting. Three grains were hand planted in each hill. Thinning to one plant per hill was done before the first irrigation. Hoeing twice was done for controlling weeds before the first and second irrigations. Nitrogen fertilizer in the form of urea $(46.6 \% \mathrm{~N})$, was applied in two equal doses before the first and the second irrigations, respectively. Recommended pest control was applied when necessary.

\section{Experimental Design}

A split-split plot design with four replications was conducted where maize hybrids were distributed in main plots, nitrogen fertilizer levels were allocated in sub-plots, while phosphorus fertilizer levels were kept in sub sub-plots according to Steel and Torrie (1980).

\section{Soil Sampling and Analysis}

Soil samples from the experimental locations were obtained from the upper $30 \mathrm{~cm}$ soil surface during land preparation in both 2014 and 2015 seasons. The soil had normal percentage of salinity and its drainage was naturally well. Both physical and chemical analyses of the soil were carried out by the methods described by Jackson (1958). Whereas N, P and K elements as well as some micronutrients were determined by applying the procedure documented by Lindsay and Norvell (1978). The soil of the experimental site was clay in texture where it had a particle size distribution of $21.98,31.85$ and $46.17 \%$ for sand, silt and clay, respectively. The soil had an average $\mathrm{pH}$ of 8.05 . The average available N,P and $\mathrm{K}$ contents were $30.79,6.01$ and $121.0 \mathrm{ppm}$ respectively (average of the two seasons).

\section{Studied Characters}

1. Days to $50 \%$ tasseling.

2. Days to $50 \%$ silking.

3. Chlorophyll content.
Five SPAD-503 readings were measured at 70 days after planting on ear-bearing leaves of five plants by the portable chlorophyll meter (SPAD-503, Minolta, Japan). Mean of five SPAD-503 readings were recorded. Chlorophyll content was determined as SPAD unit, these units were transformed to $\mathrm{mg} \mathrm{m}^{-2}$ as described by Monje and Bugbee (1992) as follows: (Chlorophyll content $=80.05+10.4$ [SPAD 503]).

4. Plant height $(\mathrm{cm})$.

5. Ear height $(\mathrm{cm})$.

6. Ear leaf area: It was measured as described by Mckee, 1964. ELA $=$ Leaf $_{\text {Length }} \times$ Leaf width $\times 0.75$.

7. Leaf area index (LAI): It was measured as described by Watson (1952). LAI = leaf area/ plant/ land area (at 70 days after planting).

8. Relative photosynthetic potential for grain yield/ plant. $=$ Grain yield (g./plant) $/$ LAI.

9. Relative photosynthetic potential for stover yield $/$ plant. $=$ Stover yield (g./plant) $/$ LAI.

10. Relative photosynthetic potential for biological yield $(\mathrm{g} . / \mathrm{plant}) .=[$ Grain yield $(\mathrm{g} . / \mathrm{plant})+$ Stover yield (g./ plant)] / LAI.

11. Ear length $(\mathrm{cm})$.

12. Ear diameter $(\mathrm{cm})$.

13. Stem diameter (cm.).

14. Number of rows/ear.

15. Number of grains/row.

16. 100 - grain weight (g).

17. Grain yield (g./plant).

18. Stover yield (g./plant).

19. Biological yield (g./plant).

20. Grain yield (ardab /fad.).

21. Biological yield (ton /fad.).

22. Ear leaf efficiency $=$ Grain yield (g./plant) / Ear leaf area.

23. Migration coefficient $=$ Ear dry weight at harvest / total plant dry weight at harvest. 


\section{Statistical Analysis}

Data were statically analyzed according to Gomez and Gomez (1984), using the computer MSTAT-C statistical analysis package Freed et al. (1989). Treatment means were compared according to the LSD test. In the tables of the analysis of variance $* * *$ indicate significant at 0.05 and 0.01 levels of probability, respectively as described by Waller and Duncan (1969).

\section{RESULTS AND DISCUSSION}

\section{Days to $50 \%$ Tasseling, Days to $50 \%$ Silking and Chlorophyll Content}

\section{Maize hybrid differences}

The results confirmed highly significant differences between the two maize hybrids concerning days to $50 \%$ tasseling, days to $50 \%$ silking and chlorophyll content in both seasons and their consolidated data with the exception to the first season of chlorophyll content (Table 1). The results indicated clearly that the maize cultivar hybrid SC. 128 was always superior in each the aforementioned characters. This was completely true in both seasons and their combined analysis. The differences in these characters between the two maize hybrids might be attributed to the genetically variation. These results are in line with those reported by Mahgoub and El-Shenawy (2006), Attia et al. (2009) and Mukhtar et al. (2012).

\section{Nitrogen level effect}

It is quite clear from Table 1 that application of $\mathrm{N}$ fertilizer was without significant effect on days to $50 \%$ tasseling and days to $50 \%$ silking in the second season. But, in the first season and combined data, the differences had no clear trend for these characters. Regarding chlorophyll content, each increase of $\mathrm{N}$ level up to $135 \mathrm{~kg} \mathrm{~N} /$ fad., reflected significant increase in both seasons and their combined. These results suggest that nitrogen favored growth of maize plant. These results are in harmony with the findings of El-Murshedy (2002), El-Azab (2012), Darwich (2013) and El-Sobky (2014) as they found that maize reached tassling and silking later when well nitrogen fertilization took place compared with the low nitrogen fertilized ones.

\section{Phosphorus level effect}

Application of $\mathrm{P}$ fertilizer was without significant effect on both days to $50 \%$ tasseling and days to $50 \%$ silking in both seasons and their consolidated data. Regarding chlorophyll content, each increase of $\mathrm{P}$ level up to $31 \mathrm{~kg}$ $\mathrm{P}_{2} \mathrm{O}_{5} /$ fad., reflected as significant increase in both seasons and their combined, but the first $\mathrm{P}$ increment was quite enough to increase the chlorophyll content significantly in the first season.

\section{Interaction Effect}

Although the days to $50 \%$ tassling was not significantly affected by $\mathrm{P}$ application, yet it had a significant affect to $\mathrm{P}$ through the $\mathrm{N}$ and $\mathrm{P}$ interaction (Table 1-b). In general, under checks or $31 \mathrm{~kg} \mathrm{P}_{2} \mathrm{O}_{5} /$ fad., the check of $\mathrm{N}$ fertilizer had higher days to $50 \%$ tasseling, but under $15.5 \mathrm{~kg}$ $\mathrm{P}_{2} \mathrm{O}_{5} /$ fad., the four $\mathrm{N}$ levels had the same days to $50 \%$ tasseling. Under check of $\mathrm{N}$ and $\mathrm{P}$ fertilizer, days to $50 \%$ tasseling had the highest value (57.38 days). This was also observed in days to $50 \%$ silking. Therefor, the highest days to $50 \%$ silking ( 58.33 days) was recorded when $\mathrm{N}$ and $\mathrm{P}$ didn't added to maize plants.

Under checks or $15.5 \mathrm{~kg} \mathrm{P}_{2} \mathrm{O}_{5} /$ fad., any increment in $\mathrm{N}$ level up to $90 \mathrm{~kg} \mathrm{~N} /$ fad., was acompanied with significant increase in chlorophyll content while under $31 \mathrm{~kg} \mathrm{P}_{2} \mathrm{O}_{5} /$ fad., the high $\mathrm{N}$ level continued to increase significantly chlorophyll content up to $135 \mathrm{~kg}$ $\mathrm{N} /$ fad. Under any $\mathrm{N}$ level chlorophyll content significantly increased with $\mathrm{P}$ application of 31 $\mathrm{kg} \mathrm{P}_{2} \mathrm{O}_{5} /$ fad.

It is evident from Table 1-a that under $\mathrm{N}$ level of $90 \mathrm{~kg} \mathrm{~N} / \mathrm{fad}$., chlorophyll content of maize hybrid SC.128 was higher than that of SC.176 hybrids. Under the other N levels both maize hybrids SC.128 and SC. 176 had the same chlorophyll content. 
Zagazig J. Agric. Res., Vol. 44 No. (1) 2017

Table 1. Days to 50\% tasseling, days to $50 \%$ silking and Chlorophyll content $\left(\mathrm{mg} \mathrm{m}^{-2}\right)$ of the two maize hybrids as affected by nitrogen, phosphorus fertilizer levels and their interactions in 2014, 2015 seasons and their combined data

\begin{tabular}{|c|c|c|c|c|c|c|c|c|c|}
\hline \multirow[t]{2}{*}{$\begin{array}{l}\text { Main effects and } \\
\text { interactions }\end{array}$} & \multicolumn{3}{|c|}{$\begin{array}{l}\text { Days to } 50 \% \\
\text { tasseling }\end{array}$} & \multicolumn{3}{|c|}{$\begin{array}{l}\text { Days to 50\% } \\
\text { silking }\end{array}$} & \multicolumn{3}{|c|}{$\begin{array}{c}\text { Chlorophyll content } \\
\left(\mathrm{mg} \mathrm{m}^{-2}\right)\end{array}$} \\
\hline & 2014 & 2015 & Comb. & 2014 & 2015 & Comb. & 2014 & 2015 & Comb. \\
\hline \multicolumn{10}{|l|}{ Maize hybrid (H) } \\
\hline SC 128 & $56.96 \mathrm{a}$ & $57.19 \mathrm{a}$ & $57.07 \mathrm{a}$ & $58.21 \mathrm{a}$ & $57.98 \mathrm{a}$ & $58.09 \mathrm{a}$ & 570.03 & $605.66 \mathrm{a}$ & $587.84 \mathrm{a}$ \\
\hline SC 176 & $55.08 \mathrm{~b}$ & $56.15 \mathrm{~b}$ & $55.62 \mathrm{~b}$ & $56.19 \mathrm{~b}$ & $57.13 \mathrm{~b}$ & $56.66 \mathrm{~b}$ & 557.70 & $587.01 \mathrm{~b}$ & $572.35 \mathrm{~b}$ \\
\hline F. test & $* *$ & $* *$ & $* *$ & $* *$ & $* *$ & $* *$ & NS & $* *$ & $* *$ \\
\hline \multicolumn{10}{|c|}{ Nitrogen level (kg N/fad) $N$} \\
\hline $\mathbf{0}$ & $56.46 \mathrm{a}$ & 57.17 & $56.81 \mathrm{a}$ & $57.58 \mathrm{a}$ & 58.08 & $57.83 \mathrm{a}$ & $434.08 \mathrm{~d}$ & $1469.21 \mathrm{~d}$ & $451.64 \mathrm{~d}$ \\
\hline 45 & $56.29 \mathrm{a}$ & 56.54 & $56.42 \mathrm{a}$ & $57.58 \mathrm{a}$ & 57.46 & $57.52 \mathrm{a}$ & $559.18 \mathrm{c}$ & $587.54 \mathrm{c}$ & $573.36 \mathrm{c}$ \\
\hline 90 & $55.58 \mathrm{~b}$ & 56.25 & $55.92 \mathrm{~b}$ & $56.71 \mathrm{~b}$ & 57.08 & $56.90 \mathrm{~b}$ & $622.15 \mathrm{~b}$ & $647.81 \mathrm{~b}$ & $634.98 \mathrm{~b}$ \\
\hline 135 & $55.75 \mathrm{~b}$ & 56.71 & $56.23 \mathrm{ab}$ & $56.92 \mathrm{~b}$ & 57.58 & $57.25 \mathrm{a}$ & $640.04 a$ & $680.77 \mathrm{a}$ & $660.41 \mathrm{a}$ \\
\hline F. test & $* *$ & NS & $* *$ & $* *$ & NS & $* *$ & $* *$ & $* *$ & $* *$ \\
\hline \multicolumn{10}{|c|}{ Phosphorus level (kg. $\mathrm{P}_{2} \mathrm{O}_{5} /$ fad.) P } \\
\hline $\mathbf{0}$ & 56.25 & 56.63 & 56.44 & 57.38 & 57.53 & 57.45 & $542.38 \mathrm{~b}$ & $567.78 \mathrm{c}$ & $555.08 \mathrm{c}$ \\
\hline 15.5 & 55.88 & 56.75 & 56.31 & 57.13 & 57.56 & 57.34 & $572.50 \mathrm{a}$ & $599.58 \mathrm{~b}$ & $586.04 \mathrm{~b}$ \\
\hline 31 & 55.94 & 56.63 & 56.28 & 57.09 & 57.56 & 57.33 & $576.71 a$ & $621.65 \mathrm{a}$ & $599.18 \mathrm{a}$ \\
\hline F. test & NS & NS & NS & NS & NS & NS & $* *$ & $* *$ & $* *$ \\
\hline \multicolumn{10}{|l|}{ Interactions } \\
\hline $\mathbf{H} \times \mathbf{N}$ & $*$ & NS & NS & $*$ & NS & NS & $*$ & $*$ & $* *$ \\
\hline $\mathbf{H} \times \mathbf{P}$ & NS & NS & NS & NS & NS & NS & $* *$ & NS & NS \\
\hline $\mathbf{N} \times \mathbf{P}$ & ** & NS & $* *$ & $*$ & NS & * & ** & * & $* *$ \\
\hline
\end{tabular}

*** indicate significant at 0.05 and 0.01 levels of probability, respectively. $\mathrm{NS}=$ Not significant.

Table 1-a. Effect of interaction between maize hybrids and nitrogen fertilizer levels on chlorophyll content $\left(\mathrm{mg} \mathrm{m}^{-2}\right)$ (combined)

\begin{tabular}{lcccc}
\hline $\begin{array}{l}\text { Maize hybrid } \\
\text { (H) }\end{array}$ & \multicolumn{4}{c}{ Chlorophyll $\left(\mathbf{m g ~ m}^{-2}\right)$} \\
\cline { 2 - 5 } & $\mathbf{0}$ & $\mathbf{4 5}$ N level (kg N/ fad.) \\
\cline { 2 - 5 } & $\mathrm{C}$ & $\mathrm{B}$ & $\mathrm{90}$ & $\mathbf{1 3 5}$ \\
\hline \multirow{2}{*}{ SC 128 } & $446.82 \mathrm{a}$ & $583.76 \mathrm{a}$ & $654.29 \mathrm{a}$ & $666.50 \mathrm{a}$ \\
& $\mathrm{D}$ & $\mathrm{C}$ & $\mathrm{B}$ & $\mathrm{A}$ \\
SC 176 & $456.47 \mathrm{a}$ & $562.96 \mathrm{a}$ & $615.67 \mathrm{~b}$ & $654.32 \mathrm{a}$ \\
\hline
\end{tabular}


Table 1-b. Effect of interaction between nitrogen fertilizer levels and phosphorus fertilizer levels on days to $50 \%$ tasseling, days to $50 \%$ silking and chlorophyll content $\left(\mathrm{mg} \mathrm{m}^{-2}\right)$ (combined)

\begin{tabular}{|c|c|c|c|c|c|c|c|c|c|}
\hline \multirow{3}{*}{$\begin{array}{l}\text { N level } \\
\text { (kg N/fad.) }\end{array}$} & \multicolumn{3}{|c|}{ Days to $50 \%$ tasseling } & \multirow{2}{*}{\multicolumn{3}{|c|}{$\begin{array}{l}\text { Days to } 50 \% \text { silking } \\
\text { P level }\left(\mathrm{kg} \mathrm{P}_{2} \mathrm{O}_{5} / \text { fad. }\right)\end{array}$}} & \multicolumn{3}{|c|}{ Chlorophyll ( $\left.\mathrm{mg} \mathrm{m}^{-2}\right)$} \\
\hline & & & & & & & & & \\
\hline & $\mathbf{0}$ & 15.5 & 31 & $\mathbf{0}$ & 15.5 & 31 & $\mathbf{0}$ & $\mathbf{1 5 . 5}$ & 31 \\
\hline \multirow{3}{*}{ 0 } & A & B & $\mathrm{AB}$ & A & B & $\mathrm{AB}$ & $\mathrm{C}$ & B & A \\
\hline & $57.38 \mathrm{a}$ & $56.20 \mathrm{a}$ & $56.83 \mathrm{a}$ & $58.33 \mathrm{a}$ & $57.44 \mathrm{a}$ & $57.75 \mathrm{a}$ & 426.17 & 453.14 & $475.62 d$ \\
\hline & A & A & A & A & A & A & B & A & A \\
\hline \multirow[t]{2}{*}{45} & $56.53 \mathrm{ab}$ & $56.38 \mathrm{a}$ & $56.33 \mathrm{ab}$ & $57.63 \mathrm{a}$ & $57.44 \mathrm{a}$ & $57.50 \mathrm{ab}$ & 531.77 & $601.78 \mathrm{l}$ & $586.55 \mathrm{c}$ \\
\hline & A & A & $\mathrm{A}$ & A & A & A & B & $\mathrm{AB}$ & A \\
\hline \multirow[t]{2}{*}{90} & $55.88 \mathrm{~b}$ & $56.19 \mathrm{a}$ & $55.69 \mathrm{~b}$ & $56.83 \mathrm{~b}$ & $57.19 \mathrm{a}$ & $56.69 \mathrm{~b}$ & 622.54 & $633.67 a$ & $648.74 \mathrm{~b}$ \\
\hline & A & A & A & A & A & A & $\mathrm{B}$ & $\mathrm{B}$ & A \\
\hline 135 & $55.94 \mathrm{ab}$ & $56.44 \mathrm{a}$ & $56.33 \mathrm{ab}$ & $57.03 \mathrm{ab}$ & $57.31 \mathrm{a}$ & $57.38 \mathrm{ab}$ & 639.85 & $655.56 a$ & $685.81 \mathrm{a}$ \\
\hline
\end{tabular}

\section{Plant and Ear heights and Ear Leaf Area}

\section{Maize hybrid differences}

Table 2 shows plant and ear heights and ear leaf area of the two maize hybrids as affected by $\mathrm{N}$ and $\mathrm{P}$ fertilizer levels in the two seasons and their combined.

It is evident from Table 2 that plant height of the two maize hybrids under study significantly varied in the two seasons and their combined while ear height varied in the combined only. According to the combined of the two seasons, SC. 176 had taller plants $\left(246.5 \mathrm{~cm}^{2}\right)$ with smaller ear leaf area $\left(598.6 \mathrm{~cm}^{2}\right)$ than SC. 128 which had shorter plants $(241.1 \mathrm{~cm})$ with larger ear leaf area $\left(668.9 \mathrm{~cm}^{2}\right)$.

Significant hybrid differences were reported in the literature regarding plant height and ear height by Khalil (2001) and Mowafy (2003) as well as Mahgoub and El-Shenawy (2006) and ear leaf area Oraby et al. (2005). These variation were attributed to differences in the genetic back ground of the studied hybrids.

\section{Nitrogen level effect}

Results in Table 2 reveal that increasing nitrogen levels significantly increased maize plant height in the two seasons and their combined analysis. Based on the combined, adding 45, 90 and $135 \mathrm{~kg} \mathrm{~N} /$ fad., increased plant height by $10.01,14.70,19.40 \%$, respectively. Regarding both ear height and ear leaf area, they were significantly increased due to the addition of $90 \mathrm{~kg} \mathrm{~N} / \mathrm{fad}$. The increased of $\mathrm{N}$ levels from 90 to $135 \mathrm{~kg} /$ fad., failed to reflect significant increase in both characters.

The elongating effect of $\mathrm{N}$ is well crown and is documented in maize by Toaima and Saleh (2003), Abd-Alla (2005), Soliman and Gharib (2011), Darwich (2013), El-Sobky (2014), Olusegun (2015) and Seadh et al. (2015) reported significant increase in plant height and ear height of maize due to addition 90 and 140 $\mathrm{kg} \mathrm{N} /$ fad., respectively. Similar results were reported regarding ear leaf area by Darwich (2013) and El-Sobky (2014) when they added 60 and $120 \mathrm{~kg} \mathrm{~N} /$ fad., respectively.

\section{Phosphors level effect}

It is evident from Table 2 that each $\mathrm{P}$ increment up to $31 \mathrm{~kg} \mathrm{P}_{2} \mathrm{O}_{5} /$ fad., produced significant increase in each of plant height, ear height and ear leaf area in both seasons and their combined. Based on the combined data, adding 15.5 and $31 \mathrm{~kg} \mathrm{P}_{2} \mathrm{O}_{5} /$ fed., increased ear leaf area by 11.08 and $17.89 \%$, receptively. These results are similar to those proved by Mukhtar et al. (2011), Khan et al. (2014) and Olusegun (2015).

\section{Interaction effect}

Generally, maize plant height exhibited more response to phosphorus fertilizer when $\mathrm{N}$ fertilizer was added by either 90 or $135 \mathrm{~kg}$ $\mathrm{N} /$ fad. Under check or $45 \mathrm{~kg} \mathrm{~N} /$ fad., plant height was only increased by $15.5 \mathrm{~kg} \mathrm{P}_{2} \mathrm{O}_{5} /$ fad. While 
Zagazig J. Agric. Res., Vol. 44 No. (1) 2017

Table 2. Plant height $(\mathrm{cm})$, ear height $(\mathrm{cm})$ and ear leaf area $\left(\mathrm{cm}^{2}\right)$ of the two maize hybrids as affected by nitrogen fertilizer levels, phosphorus fertilizer levels and their interactions in 2014, 2015 seasons and their combined data

\begin{tabular}{|c|c|c|c|c|c|c|c|c|c|}
\hline \multirow{2}{*}{$\begin{array}{l}\text { Main effects and } \\
\text { interactions }\end{array}$} & \multicolumn{3}{|c|}{ Plant height (cm) } & \multicolumn{3}{|c|}{ Ear height (cm) } & \multicolumn{3}{|c|}{ Ear leaf area } \\
\hline & 2014 & 2015 & Comb. & 2014 & 2015 & Comb. & 2014 & 2015 & $\overline{\text { Comb. }}$ \\
\hline \multicolumn{10}{|l|}{ Maize hybrid (H) } \\
\hline SC 128 & $233.77 b$ & $248.51 \mathrm{~b}$ & $241.14 \mathrm{~b}$ & 117.83 & 131.29 & \multicolumn{4}{|c|}{124.56 b 658.41 a 679.47 a 668.94 a } \\
\hline SC 176 & $242.40 \mathrm{a}$ & $250.69 a$ & $246.54 \mathrm{a}$ & 125.35 & 133.26 & \multicolumn{4}{|c|}{129.31 a 596.54 b 600.65 b 598.59 b } \\
\hline F. test & $* *$ & $* *$ & $* *$ & NS & NS & $* *$ & $* *$ & $* *$ & $* *$ \\
\hline \multicolumn{10}{|c|}{ Nitrogen level (kg N/fad.) N } \\
\hline $\mathbf{0}$ & \multicolumn{9}{|c|}{$217.17 \mathrm{~d} 221.98$ d 219.58 d 112.54 b 119.28 c 115.91 c 486.52 c 491.07 c 488.79 c } \\
\hline 45 & \multicolumn{9}{|c|}{$235.92 \mathrm{c} 247.33$ c 241.62 c 118.88 b 130.91 b 124.89 b 610.49 b 611.63 b 611.06 b } \\
\hline 90 & \multicolumn{9}{|c|}{244.33 b 259.33 b 251.83 b 126.50 a 140.03 a 133.27 a 699.64 a 716.30 a 707.97 a } \\
\hline 135 & \multicolumn{9}{|c|}{254.90 a 269.77 a 262.34 a 128.46 a 138.87 a 133.66 a 713.24 a 741.23 a 727.23 a } \\
\hline F. test & $* *$ & ** & $* *$ & $* *$ & $* *$ & $* *$ & $* *$ & $* *$ & $* *$ \\
\hline \multicolumn{10}{|c|}{ Phosphorus level (kg. $\mathbf{P}_{2} \mathrm{O}_{5} /$ fad.) P } \\
\hline $\mathbf{0}$ & \multicolumn{9}{|c|}{226.56 c 234.29 c 230.43 c 118.19 c 126.99 c 122.59 c 572.46 c 584.07 c 578.27 c } \\
\hline 15.5 & \multicolumn{9}{|c|}{$238.84 b 251.45$ b 245.15 b 121.00 b 131.64 b 126.32 b 634.57 b 650.31 b 642.44 b } \\
\hline 31 & \multicolumn{9}{|c|}{ 248.84a 263.06 a 255.95 a 125.59 a 138.19 a 131.89 a 675.39 a 685.79 a 680.59 a } \\
\hline F. test & $* *$ & ** & $* *$ & & $* *$ & $* *$ & $* *$ & $* *$ & $* *$ \\
\hline \multicolumn{10}{|l|}{ Interactions } \\
\hline $\mathbf{H} \times \mathbf{N}$ & NS & $* *$ & $*$ & NS & NS & $* *$ & $*$ & NS & $* *$ \\
\hline $\mathbf{H} \times \mathbf{P}$ & NS & NS & NS & NS & NS & NS & NS & NS & NS \\
\hline $\mathbf{N} \times \mathbf{P}$ & $* *$ & $*$ & $* *$ & NS & $*$ & $* *$ & NS & $*$ & $* *$ \\
\hline
\end{tabular}

*** indicate significant at 0.05 and 0.01 levels of probability, respectively. $\mathrm{NS}=$ Not significant.

under the two high levels of N (90 and 135 $\mathrm{kg} /$ fad.), plant height increased with increased $\mathrm{P}$ up to $31 \mathrm{~kg} \mathrm{P}_{2} \mathrm{O}_{5} /$ fad., (Table 2-b).

Under unfertilized plants with of $\mathrm{P}$, ear height was slightly affected by $\mathrm{N}$ level up to application of $135 \mathrm{~kg} \mathrm{~N} / \mathrm{fad}$. However under both 15.5 and $31 \mathrm{~kg} \mathrm{P}_{2} \mathrm{O}_{5} /$ fad., that height increased by increasing $\mathrm{N}$ level up to $90 \mathrm{~kg}$ $\mathrm{N} /$ fad. Under $45 \mathrm{~kg} \mathrm{~N} /$ fad., there were no significant differences between the three $\mathrm{P}$ levels.

In general, maize ear leaf area reported more response to phosphors fertilizer when plants were fertilized by $135 \mathrm{~kg} \mathrm{~N} / \mathrm{fad}$., whereas under the other three $\mathrm{N}$ levels ear leaf area increased up to $15.5 \mathrm{~kg} \mathrm{P}_{2} \mathrm{O}_{5} /$ fad. Therefore, the largest ear leaf area (780.83) was recorded when maize was fertilized by $31 \mathrm{~kg} \mathrm{P}_{2} \mathrm{O}_{5} /$ fad., and $135 \mathrm{~kg} \mathrm{~N} / \mathrm{fad}$.

It is evident from Table 2-a that under fertilizing maize plant by either 45 or $90 \mathrm{~kg}$ $\mathrm{N} /$ fad., plant height of SC. 176 was higher than that of SC. 128 hybrids. In this respect it is noticed that plant height of SC. 128 maize variety responded to $\mathrm{N}$ more than maize variety SC. 176 as plant height value increased consistently with $\mathrm{N}$ in the first case but only increased till $45 \mathrm{~kg} \mathrm{~N} /$ fad., in SC. 176. Also ear leaf area of both SC. 128 and SC. 176 responded to $\mathrm{N}$ up to $90 \mathrm{~kg} \mathrm{~N} / \mathrm{fad}$., whereas SC. 128 had higher ear leaf area than SC. 176 under different nitrogen levels. 
Table 2-a. Effect of interaction between maize hybrids and nitrogen fertilizer levels on plant height $(\mathrm{cm})$, ear height $(\mathrm{cm})$ and ear leaf area (combined)

\begin{tabular}{|c|c|c|c|c|c|c|c|c|c|c|c|c|}
\hline \multirow{3}{*}{$\begin{array}{l}\text { Maize hybrid } \\
\text { (H) }\end{array}$} & \multicolumn{4}{|c|}{ Plant height (cm) } & \multicolumn{4}{|c|}{ Ear height (cm) } & \multicolumn{4}{|c|}{ Ear leaf area } \\
\hline & \multicolumn{12}{|c|}{ N level (kg N/ fad.) } \\
\hline & $\mathbf{0}$ & 45 & 90 & 135 & $\mathbf{0}$ & 45 & 90 & 135 & $\mathbf{0}$ & 45 & 90 & 135 \\
\hline \multirow{3}{*}{ SC 128} & $\mathrm{D}$ & $\mathrm{C}$ & $\mathrm{B}$ & $\mathrm{A}$ & B & $\mathrm{AB}$ & $\mathrm{AB}$ & A & $\mathrm{C}$ & B & A & A \\
\hline & $221.95 \mathrm{a}$ & $234.43 b$ & $246.91 b$ & $261.28 \mathrm{a}$ & $114.34 \mathrm{a}$ & $121.41 \mathrm{a}$ & $127.68 \mathrm{~b}$ & 134.83 & 539.19 & 657.72 & 730.58 & $748.26 \mathrm{a}$ \\
\hline & $\mathrm{C}$ & $\mathrm{AB}$ & $\mathrm{A}$ & $\mathrm{A}$ & $\mathrm{B}$ & $\mathrm{AB}$ & A & $\mathrm{AB}$ & $\mathrm{C}$ & B & A & A \\
\hline SC 176 & $217.20 \mathrm{a}$ & $248.81 \mathrm{a}$ & $256.75 \mathrm{a}$ & $263.41 \mathrm{a}$ & $117.50 \mathrm{a}$ & $128.38 \mathrm{a}$ & $138.86 \mathrm{a}$ & $132.49 \mathrm{a}$ & 438.40 & $564.40 \mathrm{l}$ & $685.36 \mathrm{~b}$ & $706.21 \mathrm{~b}$ \\
\hline
\end{tabular}

Table 2-b. Effect of interaction between nitrogen fertilizer levels and phosphorus fertilizer levels on plant height $(\mathrm{cm})$, ear height $(\mathrm{cm})$ and ear leaf area (combined)

\begin{tabular}{|c|c|c|c|c|c|c|c|c|c|}
\hline \multirow{3}{*}{$\begin{array}{l}\text { N level } \\
\text { (kg N/fad.) }\end{array}$} & \multicolumn{3}{|c|}{ Plant height (cm) } & \multicolumn{3}{|c|}{ Ear height (cm) } & \multicolumn{3}{|c|}{ Ear leaf area } \\
\hline & \multicolumn{9}{|c|}{ P level (kg $\mathrm{P}_{2} \mathrm{O}_{5} /$ fad.) } \\
\hline & $\mathbf{0}$ & 15.5 & 31 & $\mathbf{0}$ & 15.5 & 31 & $\mathbf{0}$ & 15.5 & 31 \\
\hline & $\mathrm{B}$ & A & A & $\mathrm{B}$ & B & A & $\mathrm{B}$ & A & A \\
\hline \multirow[t]{2}{*}{$\mathbf{0}$} & $210.78 \mathrm{~b}$ & $220.14 \mathrm{c}$ & $227.81 \mathrm{~d}$ & $109.91 \mathrm{~b}$ & $115.38 \mathrm{~b}$ & $122.45 \mathrm{~b}$ & $407.13 \mathrm{c}$ & $512.98 \mathrm{c}$ & $546.27 \mathrm{c}$ \\
\hline & $\mathrm{B}$ & A & A & A & A & A & $\mathrm{B}$ & A & A \\
\hline \multirow[t]{2}{*}{45} & $232.66 \mathrm{ab}$ & $242.79 \mathrm{~b}$ & $249.41 \mathrm{c}$ & $124.68 \mathrm{a}$ & $122.38 \mathrm{~b}$ & $127.63 \mathrm{~b}$ & $577.28 \mathrm{~b}$ & $609.21 \mathrm{~b}$ & $646.69 \mathrm{~b}$ \\
\hline & $\mathrm{C}$ & $\mathrm{B}$ & $\mathrm{A}$ & $\mathrm{AB}$ & $\mathrm{B}$ & $\mathrm{A}$ & $\mathrm{B}$ & A & A \\
\hline \multirow[t]{2}{*}{90} & $238.23 \mathrm{a}$ & $251.35 \mathrm{~b}$ & $265.91 \mathrm{~b}$ & $131.18 \mathrm{a}$ & $131.05 \mathrm{a}$ & $137.58 \mathrm{a}$ & $663.92 \mathrm{a}$ & $711.43 \mathrm{a}$ & $748.56 \mathrm{a}$ \\
\hline & $\mathrm{C}$ & $\mathrm{B}$ & $\mathrm{A}$ & $\mathrm{B}$ & A & A & $\mathrm{C}$ & $\mathrm{B}$ & A \\
\hline 135 & $240.04 \mathrm{a}$ & $266.31 \mathrm{a}$ & $280.68 \mathrm{a}$ & $124.60 \mathrm{a}$ & $136.48 \mathrm{a}$ & $139.91 \mathrm{a}$ & $664.73 \mathrm{a}$ & $736.14 \mathrm{a}$ & $780.83 \mathrm{a}$ \\
\hline
\end{tabular}

\section{Maize photosynthetic attributes}

Effects of nitrogen and phosphorus levels on each of leaf area index (LAI), relative photosynthetic for grain yield/plant, relative photosynthetic for stover yield/plant and relative photosynthetic for biological yield/plant of two maize hybrids are given in Table 3.

\section{Maize hybrids differences}

The results in Table 3 shows that the two maize hybrids under study significantly varied in each of LAI and relative photosynthetic potential for grain and stover yields/ plant as indicated from the combined analysis.
Insignificant differences between the two maize hybrids on relative photosynthetic potential for biological yield/plant in the two seasons and their combined were detected. In the both seasons and their combined SC.128 had larger LAI and higher relative photosynthetic potential of stover yield/plant than SC.176 which had higher relative photosynthetic potential for grain yield/plant in combined analysis.

In the literature several author, reported significant cultivar differences in leaf area index and photosynthetic attributes such as Khalil (2001), El-Nagar (2002) and Atia (2006). 
Table 3. Leaf area index (LAI), Relative photosynthetic potential for grain yield/ plant, stover yield/ plant and biological yield/ plant of the two maize hybrids as affected by nitrogen and phosphorus fertilizer levels and their interactions in 2014, 2015 seasons and their combined data

\begin{tabular}{|c|c|c|c|c|c|c|c|c|c|c|c|c|}
\hline \multirow{3}{*}{$\begin{array}{l}\text { Main effects and } \\
\text { interactions }\end{array}$} & \multirow{2}{*}{\multicolumn{3}{|c|}{$\begin{array}{l}\text { Leaf area index } \\
\text { (LAI) }\end{array}$}} & \multicolumn{9}{|c|}{$\begin{array}{l}\text { Relative photosynthetic potential for } \\
\end{array}$} \\
\hline & & & & \multicolumn{3}{|c|}{ Grain yield/plant } & \multicolumn{3}{|c|}{ Stover yield/ plant } & \multicolumn{3}{|c|}{ Biological yield/ plant } \\
\hline & 2014 & 2015 & Comb. & 2014 & 2015 & Comb. & 2014 & 2015 & Comb. & 2014 & 2015 & Comb. \\
\hline \multicolumn{13}{|l|}{ Maize hybrid (H) } \\
\hline SC 128 & $4.30 \mathrm{a}$ & $4.52 \mathrm{a}$ & $4.41 \mathrm{a}$ & $31.61 \mathrm{~b}$ & 33.02 & $32.32 b$ & $73.41 \mathrm{a}$ & $71.26 \mathrm{a}$ & $72.34 \mathrm{a}$ & 105.03 & 104.28 & 104.65 \\
\hline SC 176 & $3.64 \mathrm{~b}$ & $3.81 \mathrm{~b}$ & $3.72 \mathrm{~b}$ & $34.08 \mathrm{a}$ & 35.83 & $34.95 \mathrm{a}$ & $70.12 b$ & $66.68 b$ & $68.40 \mathrm{~b}$ & 104.20 & 102.51 & 103.35 \\
\hline F. test & $* *$ & $* *$ & $* *$ & $* *$ & NS & $* *$ & $*$ & $* *$ & $* *$ & NS & NS & NS \\
\hline \multicolumn{13}{|l|}{$\begin{array}{l}\text { Nitrogen level } \\
\text { (kg N/fad.) N }\end{array}$} \\
\hline 0 & $1.93 \mathrm{~d}$ & $2.08 \mathrm{~d}$ & $2.00 \mathrm{~d}$ & 32.82 & 32.90 & 32.86 & $104.65 \mathrm{a}$ & $96.04 a$ & $100.35 a$ & $137.47 \mathrm{a}$ & $128.94 a$ & $133.20 \mathrm{a}$ \\
\hline 45 & $3.77 \mathrm{c}$ & $4.05 \mathrm{c}$ & $3.91 \mathrm{c}$ & 32.68 & 34.05 & 33.36 & $68.06 \mathrm{~b}$ & $65.01 \mathrm{~b}$ & $66.53 b$ & $100.73 b$ & $99.05 b$ & $99.89 \mathrm{~b}$ \\
\hline 90 & $4.73 \mathrm{~b}$ & $4.94 \mathrm{~b}$ & $4.83 \mathrm{~b}$ & 32.20 & 34.81 & 33.50 & $58.54 \mathrm{c}$ & $58.87 \mathrm{c}$ & $58.71 \mathrm{c}$ & $90.74 \mathrm{c}$ & $93.68 b c$ & $92.21 \mathrm{c}$ \\
\hline 135 & $5.45 \mathrm{a}$ & $5.59 \mathrm{a}$ & $5.52 \mathrm{a}$ & 33.70 & 35.94 & 34.82 & $55.80 \mathrm{c}$ & $55.98 \mathrm{c}$ & $55.89 \mathrm{~d}$ & $89.50 \mathrm{c}$ & $91.91 \mathrm{c}$ & $90.71 \mathrm{c}$ \\
\hline F. test & $* *$ & $* *$ & $* *$ & NS & NS & NS & $* *$ & $* *$ & $* *$ & $* *$ & $* *$ & $* *$ \\
\hline \multicolumn{13}{|l|}{$\begin{array}{l}\text { Phosphorus level } \\
\text { (kg. } \mathbf{P}_{2} \mathrm{O}_{5} / \text { fad.) P }\end{array}$} \\
\hline $\mathbf{0}$ & $3.48 \mathrm{c}$ & $3.55 \mathrm{c}$ & $3.52 \mathrm{c}$ & $27.43 \mathrm{c}$ & $30.32 \mathrm{c}$ & $28.87 \mathrm{c}$ & $77.80 \mathrm{a}$ & $78.17 \mathrm{a}$ & 77.99a & 105.22 & $108.49 \mathrm{a}$ & $106.86 a$ \\
\hline 15.5 & $4.09 \mathrm{~b}$ & $4.26 \mathrm{~b}$ & $4.17 \mathrm{~b}$ & $33.29 b$ & $34.91 b$ & $34.10 \mathrm{~b}$ & $69.65 b$ & $65.15 b$ & $67.40 \mathrm{~b}$ & 102.95 & $100.07 \mathrm{~b}$ & $101.51 b$ \\
\hline 31 & $4.35 \mathrm{a}$ & $4.69 \mathrm{a}$ & $4.52 \mathrm{a}$ & $37.82 \mathrm{a}$ & $38.03 a$ & $37.93 a$ & $67.84 b$ & $63.59 \mathrm{~b}$ & $65.72 b$ & 105.66 & $101.62 b$ & $103.64 b$ \\
\hline F. test & $* *$ & $* *$ & $* *$ & $* *$ & $* *$ & $* *$ & $* *$ & $* *$ & $* *$ & NS & $*$ & $*$ \\
\hline \multicolumn{13}{|l|}{ Interactions } \\
\hline $\mathbf{H} \times \mathbf{N}$ & $* *$ & $* *$ & $* *$ & $*$ & $*$ & $* *$ & $*$ & $* *$ & $* *$ & $* *$ & $* *$ & $* *$ \\
\hline $\mathbf{H} \times \mathbf{P}$ & $* *$ & $* *$ & $* *$ & $* *$ & $* *$ & $* *$ & NS & $* *$ & $* *$ & NS & $* *$ & $* *$ \\
\hline $\mathbf{N} \times \mathbf{P}$ & $* *$ & $* *$ & $* *$ & $* *$ & $*$ & $* *$ & $* *$ & NS & $* *$ & $*$ & $\mathrm{NS}$ & NS \\
\hline
\end{tabular}

*,** indicate significant at 0.05 and 0.01 levels of probability, respectively. $\mathrm{NS}=$ Not significant.

\section{Nitrogen level effect}

The increase of $\mathrm{N}$ level up to $135 \mathrm{~kg} \mathrm{~N} / \mathrm{fad}$., was accompanied by a significant increase in leaf area index, the opposite was completely true for both relative photosynthetic potential for stover yield/ plant and relative photosynthetic potential for biological yield/ plant. But this addition of $\mathrm{N}$ fertilizer was without significant effect on relative photosynthetic potential for grain yield/plant as indicated from the two seasons and their combined (Table 3 ).

Again, the results of LAI (Table 3) concerning the effect of $\mathrm{N}$ fertilizer level are identical with those of plant height (Table 2). The increase in LAI due to application of nitrogen was obtained by Kandil (2013), ElSobky (2014) and El-Kholy (2015).

\section{Phosphors level effect}

Results in Table 3 shows that increasing $\mathrm{P}$ level from zero to 15.5 and to $31 \mathrm{~kg} \mathrm{P}_{2} \mathrm{O}_{5} /$ fad., caused a gradually increase in both leaf area index and relative photosynthetic potential for grain yield/ plant, but photosynthetic potential for both stover and biological yields/ plant were decreased as indicated from the two seasons and their combined with exception the for first 
season of relative photosynthetic potential for biological yield/plant which was not affected by $\mathrm{P}$ level. According to the combined analysis first $\mathrm{P}$ increment (15.5 $\mathrm{P}_{2} \mathrm{O}_{5} \mathrm{~kg} / \mathrm{fad}$.), produced a significant increase of $18.46 \%$, whereas the seconded $\mathrm{P}$ increment $\left(31 \mathrm{P}_{2} \mathrm{O}_{5} \mathrm{~kg} /\right.$ fad. $)$, gave an increase of $28.41 \%$ in LAI. Also relative photosynthetic potential for grain yield/plant produced asignificant increase of $18.11 \%$ due to the first $\mathrm{P}$ increment, whereas the second $\mathrm{P}$ increment gave an increase of $31.38 \%$ only. These results are in line with those reported by Plenet et al. (2000).

\section{Interaction effect}

The two maize hybrids i.e. SC. 128 and SC.176 had the same LAI when maize was grown without applying nitrogen. SC.128 hybrid had larger LAI compared with SC.176 under the different nitrogen levels. Both maize hybrids responded to nitrogen fertilizer up to $135 \mathrm{~kg}$ N/fad., Table 3-a.

Maize hybrids SC. 128 and SC. 176 had the same relative photosynthetic potential (RPP) for grain yield/plant when maize was grown either without applying nitrogen or under both 90 and $135 \mathrm{~kg} \mathrm{~N} /$ fad. While SC. 176 hybrid had higher relative photosynthetic potential for grain yield/ plant compared with SC. 128 under $45 \mathrm{~kg} \mathrm{~N} / \mathrm{fad}$.

Maize hybrid SC. 128 responded to phosphorus fertilizer up to $15.5 \mathrm{~kg}_{2} \mathrm{O}_{5} /$ fad., only, while SC. 176 hybrid responded up to $31 \mathrm{~kg} \mathrm{P}_{2} \mathrm{O}_{5} / \mathrm{fad}$. Maize hybrid SC. 128 had higher LAI under the different $\mathrm{P}$ fertilizer levels. Under the highest $\mathrm{P}$ level used (31 $\mathrm{kg} \quad \mathrm{P}_{2} \mathrm{O}_{5} /$ fad.), both maize cultivars produced their highest photosynthetic potential for grain yield/plant. Also SC. 128 and SC. 176 had the same relative photosynthetic potential for grain yield/plant when maize was grow under without $\mathrm{P}$ fertilizer addition and under application of $31 \mathrm{~kg} \mathrm{P}_{2} \mathrm{O}_{5} /$ fad., however SC. 176 had higher RPP for grain yield/ plant under $15.5 \mathrm{~kg} \mathrm{P}_{2} \mathrm{O}_{5} / \mathrm{fad}$. The opposite was true for relative photosynthetic potential for stover yield/plant whereas increasing $P$ fertilizer decreased relative photosynthetic potential for stover/plant of the two hybrids. The two maize hybrids had the same relative photosynthetic potential for both stover and biological yields/plant under 15.5 and $31 \mathrm{~kg} \mathrm{P}_{2} \mathrm{O}_{5} /$ fad., but SC. 128 had the higher value when maize was grow under without addition $\mathrm{P}$ fertilizer while the same trend was observed in SC. 128 for relative photosynthetic potential biological yield/ plant, but $\mathrm{P}$ fertilizer appeared to have no effect on relative photosynthetic potential for biological yield/ plant for maize hybrid SC. 176.

It is evident from Table 3-c that each $\mathrm{P}$ increment was effective to increase significantly leaf area index under the two high $\mathrm{N}$ levels i.e. 90 and $135 \mathrm{~kg} \mathrm{~N} /$ fad., but under both without addition of $\mathrm{N}$ and addition $45 \mathrm{~kg} \mathrm{~N} /$ fad., the second $\mathrm{P}$ increment failed to increase LAI while each $\mathrm{N}$ increment was effective to increase significantly LAI at the three P fertilizer levels.

In general, relative photosynthetic potential for grain yield/plant was affected by the two $\mathrm{P}$ fertilizer increments for both check and $135 \mathrm{~kg}$ $\mathrm{N} /$ fad., whereas under $45 \mathrm{~kg} \mathrm{~N} / \mathrm{fad}$., the second $\mathrm{P}$ increment failed to increase relative photosynthetic potential for grain, while under $90 \mathrm{~kg} \mathrm{~N} / \mathrm{fad}$., this character responded to $\mathrm{P}$ up to $31 \mathrm{~kg} \mathrm{P}_{2} \mathrm{O}_{5} / \mathrm{fad}$. Under plants unfertilized with $\mathrm{P}$, relative photosynthetic potential for grain yield/plant was affected by $\mathrm{N}$ level up to $45 \mathrm{~kg}$ $\mathrm{N} /$ fad., however, under both 15.5 and $31 \mathrm{~kg}$ $\mathrm{P}_{2} \mathrm{O}_{5} /$ fad., that relative photosynthetic potential did not affected by varying $\mathrm{N}$ levels. But relative photosynthetic potential for stover decreased by increasing $\mathrm{N}$ level up to $90 \mathrm{~kg} \mathrm{~N} / \mathrm{fad}$., under the three $\mathrm{P}$ levels. This was also true under both without addition of $\mathrm{N}$ and $45 \mathrm{~kg} \mathrm{~N} / \mathrm{fad}$., but, under both the two high $\mathrm{N}$ levels, that relative photosynthetic potential did not affected by varying $\mathrm{P}$ levels.

\section{Ear Length and Diameter of Ear and Stem}

The results of ear length, ear diameter and stem diameter of the two maize hybrids as affected by $\mathrm{N}$ and $\mathrm{P}$ levels are presented in Table 4.

\section{Maize hybrid differences}

In both seasons and their combined maize hybrid SC.128 had higher ear length and stem diameter than SC.176, the increase valued $12.76 \%$ and $28.64 \%$ for the same respective order. This was also observed in ear diameter in combined data, but the difference did not reach the level of significance in the two seasons. Cultivar differences were reported by others in the literature such as Mohamed (2004), Abdou et al. (2012), Golezani and Tajbakhsh (2012) and Mukhtar et al. (2012). 
Table 3-a. Effect of interaction between maize hybrids and nitrogen fertilizer levels on leaf area index (LAI), relative photosynthetic potential for grain yield/ plant, stover yield/ plant and biological yield/plant (combined)

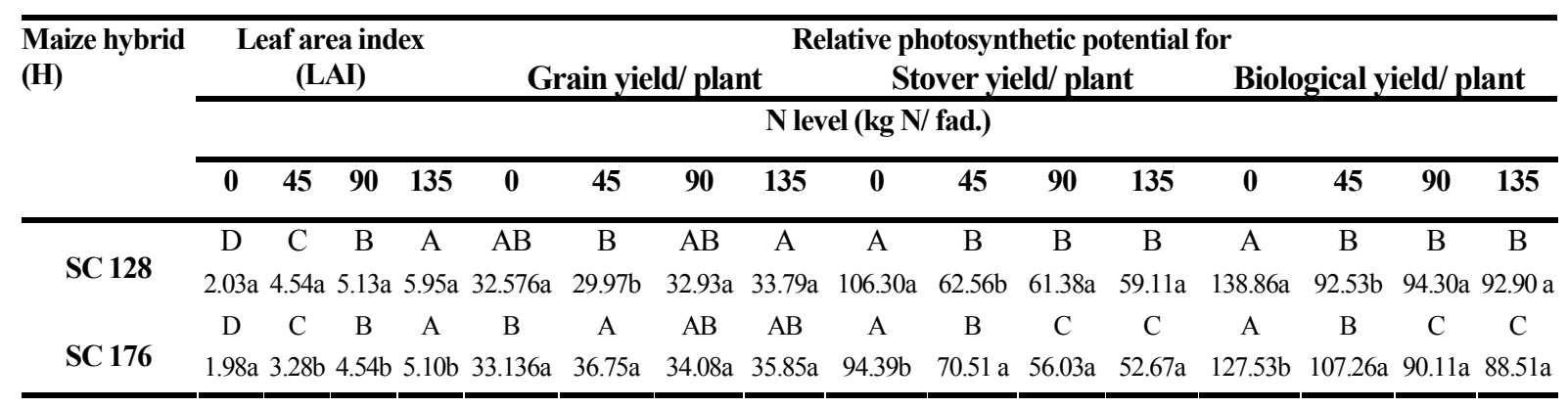

Table 3-b. Effect of interaction between maize hybrids and phosphorus fertilizer levels on leaf area index (LAI), relative photosynthetic potential for grain yield/ plant, stover yield/ plant and biological yield/ plant (combined)

\begin{tabular}{|c|c|c|c|c|c|c|c|c|c|c|c|c|}
\hline \multirow{4}{*}{$\begin{array}{l}\text { Maize hybrid } \\
\text { (H) }\end{array}$} & \multirow{2}{*}{\multicolumn{3}{|c|}{$\begin{array}{l}\text { Leaf area index } \\
\text { (LAI) }\end{array}$}} & \multicolumn{9}{|c|}{$\begin{array}{l}\text { Relative photosynthetic potential for } \\
\end{array}$} \\
\hline & & & & \multicolumn{3}{|c|}{ Grain yield/plant } & \multicolumn{3}{|c|}{ Stover yield/plant } & \multicolumn{3}{|c|}{ Biological yield/ plant } \\
\hline & \multicolumn{12}{|c|}{$P$ level $\left(k g \mathbf{P}_{2} O_{5} /\right.$ fad. $)$} \\
\hline & $\mathbf{0}$ & $\mathbf{1 5 . 5}$ & 31 & $\mathbf{0}$ & 15.5 & 31 & $\mathbf{0}$ & 15.5 & 31 & $\mathbf{0}$ & 15.5 & 31 \\
\hline SC 128 & $\begin{array}{c}\mathrm{B} \\
3.70 \mathrm{a}\end{array}$ & $\begin{array}{c}\mathrm{A} \\
4.68 \mathrm{a}\end{array}$ & $\begin{array}{c}\mathrm{A} \\
4.85 \mathrm{a}\end{array}$ & $\begin{array}{c}\mathrm{B} \\
29.33 \mathrm{a}\end{array}$ & $\begin{array}{c}\mathrm{B} \\
30.85 \mathrm{~b}\end{array}$ & $\begin{array}{c}\mathrm{A} \\
36.76 \mathrm{a}\end{array}$ & $\begin{array}{c}\mathrm{A} \\
82.74 \mathrm{a}\end{array}$ & $\begin{array}{c}\mathrm{B} \\
67.27 \mathrm{a}\end{array}$ & $\begin{array}{c}\mathrm{B} \\
66.97 \mathrm{a}\end{array}$ & $\begin{array}{c}\mathrm{A} \\
112.0 \mathrm{a}\end{array}$ & $\begin{array}{c}\mathrm{B} \\
98.14 \mathrm{a}\end{array}$ & $\begin{array}{c}\mathrm{B} \\
103.75 \mathrm{a}\end{array}$ \\
\hline SC 176 & $\begin{array}{c}\mathrm{C} \\
3.33 \mathrm{~b}\end{array}$ & $\begin{array}{c}\mathrm{B} \\
3.66 \mathrm{~b}\end{array}$ & $\begin{array}{c}\mathrm{A} \\
4.18 \mathrm{~b}\end{array}$ & $\begin{array}{c}\text { B } \\
28.42 \mathrm{a}\end{array}$ & $\begin{array}{c}\mathrm{B} \\
37.36 \mathrm{a}\end{array}$ & $\begin{array}{c}\mathrm{A} \\
39.09 \mathrm{a}\end{array}$ & $\begin{array}{c}\mathrm{A} \\
73.23 \mathrm{~b}\end{array}$ & $\begin{array}{c}\text { B } \\
67.52 \mathrm{a}\end{array}$ & $\begin{array}{c}\text { B } \\
64.45 \mathrm{a}\end{array}$ & $\begin{array}{c}\mathrm{A} \\
101.64 \mathrm{~b}\end{array}$ & $\begin{array}{c}\mathrm{A} \\
104.88 \mathrm{a}\end{array}$ & $\begin{array}{c}\mathrm{A} \\
103.54 \mathrm{a}\end{array}$ \\
\hline
\end{tabular}

Table 3-c. Effect of interaction between nitrogen fertilizer levels and phosphorus fertilizer levels on leaf area index (LAI), relative photosynthetic potential for grain yield/ plant and stover yield/ plant (combined)

\begin{tabular}{|c|c|c|c|c|c|c|c|c|c|}
\hline \multirow{4}{*}{$\begin{array}{l}\text { N level } \\
\text { (kg N/fad.) }\end{array}$} & \multirow{2}{*}{\multicolumn{3}{|c|}{$\begin{array}{c}\text { Leaf area index } \\
\text { (LAI) }\end{array}$}} & \multicolumn{6}{|c|}{ Relative photosynthetic potential for } \\
\hline & & & & \multicolumn{3}{|c|}{ Grain yield/ plant } & \multicolumn{3}{|c|}{ Stover yield/ plant } \\
\hline & \multicolumn{9}{|c|}{$P$ level (kg $\mathrm{P}_{2} \mathrm{O}_{5} /$ fad.) } \\
\hline & $\mathbf{0}$ & 15.5 & 31 & $\mathbf{0}$ & 15.5 & 31 & $\mathbf{0}$ & 15.5 & 31 \\
\hline & $\mathrm{B}$ & $\mathrm{A}$ & $\mathrm{A}$ & $\mathrm{C}$ & $\mathrm{B}$ & $\mathrm{A}$ & $\mathrm{A}$ & $\mathrm{B}$ & $\mathrm{B}$ \\
\hline $\mathbf{0}$ & $\begin{array}{c}1.55 \mathrm{~d} \\
B\end{array}$ & $\begin{array}{c}2.21 \mathrm{~d} \\
\mathrm{~A}\end{array}$ & $\begin{array}{c}2.25 \mathrm{~d} \\
\mathrm{~A}\end{array}$ & $\begin{array}{c}23.43 \mathrm{~b} \\
\mathrm{~B}\end{array}$ & $\begin{array}{c}35.06 \mathrm{a} \\
\mathrm{A}\end{array}$ & $\begin{array}{c}40.07 \mathrm{a} \\
\mathrm{A}\end{array}$ & $\begin{array}{c}116.82 \mathrm{a} \\
\mathrm{A}\end{array}$ & $\begin{array}{c}92.80 \mathrm{a} \\
\mathrm{AB}\end{array}$ & $\begin{array}{c}91.41 \mathrm{a} \\
\mathrm{B}\end{array}$ \\
\hline 45 & $\begin{array}{c}3.51 \mathrm{c} \\
\mathrm{C}\end{array}$ & $\begin{array}{c}4.07 \mathrm{c} \\
\mathrm{B}\end{array}$ & $\begin{array}{c}4.14 \mathrm{c} \\
\mathrm{A}\end{array}$ & $\begin{array}{c}30.56 \mathrm{a} \\
\mathrm{B}\end{array}$ & $\begin{array}{c}34.72 \mathrm{a} \\
\mathrm{B}\end{array}$ & $\begin{array}{c}34.81 \mathrm{~b} \\
\mathrm{~A}\end{array}$ & $\begin{array}{c}72.91 \mathrm{~b} \\
\mathrm{~A}\end{array}$ & $\begin{array}{c}65.50 \mathrm{~b} \\
\mathrm{~A}\end{array}$ & $\begin{array}{c}61.18 \mathrm{~b} \\
\mathrm{~A}\end{array}$ \\
\hline 90 & $\begin{array}{c}4.26 \mathrm{~b} \\
\mathrm{C}\end{array}$ & $\begin{array}{c}4.97 \mathrm{~b} \\
\mathrm{~B}\end{array}$ & $\begin{array}{c}5.27 \mathrm{~b} \\
\mathrm{~A}\end{array}$ & $\begin{array}{c}31.70 \mathrm{a} \\
\mathrm{C}\end{array}$ & $\begin{array}{c}31.91 \mathrm{a} \\
\mathrm{B}\end{array}$ & $\begin{array}{c}36.90 \mathrm{a} \\
\mathrm{A}\end{array}$ & $\begin{array}{c}62.46 \mathrm{c} \\
\mathrm{A}\end{array}$ & $\begin{array}{c}56.24 \mathrm{c} \\
\mathrm{A}\end{array}$ & $\begin{array}{c}57.42 \text { bc } \\
\text { A }\end{array}$ \\
\hline 135 & $4.74 \mathrm{a}$ & $5.43 \mathrm{a}$ & $6.40 \mathrm{a}$ & $29.80 \mathrm{a}$ & $34.72 \mathrm{a}$ & $39.92 \mathrm{a}$ & $59.75 \mathrm{c}$ & $55.07 \mathrm{c}$ & $52.85 \mathrm{c}$ \\
\hline
\end{tabular}


Table 4. Ear length $(\mathrm{cm})$, ear diameter $(\mathrm{cm})$ and stem diameter $(\mathrm{cm})$ of the two maize hybrids as affected by nitrogen fertilizer levels, phosphorus fertilizer levels and their interactions in 2014, 2015 seasons and their combined data

\begin{tabular}{|c|c|c|c|c|c|c|c|c|c|}
\hline \multirow{2}{*}{$\begin{array}{l}\text { Main effects and } \\
\text { interactions }\end{array}$} & \multicolumn{3}{|c|}{ Ear length (cm) } & \multicolumn{3}{|c|}{ Ear diameter $(\mathrm{cm})$} & \multicolumn{3}{|c|}{ Stem diameter $(\mathrm{cm})$} \\
\hline & 2014 & 2015 & Comb. & 2014 & 2015 & Comb. & 2014 & 2015 & Comb. \\
\hline \multicolumn{10}{|l|}{$\overline{\text { Maize hybrid (H) }}$} \\
\hline SC 128 & $20.19 \mathrm{a}$ & $23.20 \mathrm{a}$ & $21.69 \mathrm{a}$ & 4.35 & 4.56 & $4.46 \mathrm{a}$ & $2.13 \mathrm{a}$ & $2.99 \mathrm{a}$ & $2.56 \mathrm{a}$ \\
\hline SC 176 & $18.31 \mathrm{~b}$ & $20.10 \mathrm{~b}$ & $19.20 \mathrm{~b}$ & 4.18 & 4.45 & $4.31 \mathrm{~b}$ & $1.91 \mathrm{~b}$ & $2.07 \mathrm{~b}$ & $1.99 \mathrm{~b}$ \\
\hline F. test & $* *$ & $* *$ & $* *$ & NS & NS & $*$ & $* *$ & $* *$ & $* *$ \\
\hline \multicolumn{10}{|c|}{ Nitrogen level (kg N/fad.) $\mathrm{N}$} \\
\hline 0 & $15.90 \mathrm{~d}$ & $16.30 \mathrm{~d}$ & $16.10 \mathrm{~d}$ & $3.44 \mathrm{c}$ & $3.91 \mathrm{~b}$ & $3.68 \mathrm{~d}$ & $1.33 \mathrm{~d}$ & $1.79 \mathrm{~b}$ & $1.56 \mathrm{c}$ \\
\hline 45 & $19.04 \mathrm{c}$ & $21.68 \mathrm{c}$ & $20.36 \mathrm{c}$ & $4.10 \mathrm{~b}$ & $4.48 \mathrm{a}$ & $4.29 \mathrm{c}$ & $2.16 \mathrm{c}$ & $2.71 \mathrm{a}$ & $2.43 \mathrm{~b}$ \\
\hline 90 & $20.22 b$ & $22.93 \mathrm{~b}$ & $21.58 \mathrm{~b}$ & $4.60 \mathrm{a}$ & $4.75 \mathrm{a}$ & $4.67 \mathrm{~b}$ & $2.23 \mathrm{~b}$ & $2.75 \mathrm{a}$ & $2.49 \mathrm{~b}$ \\
\hline 135 & $21.84 \mathrm{a}$ & $25.68 \mathrm{a}$ & $23.76 \mathrm{a}$ & $4.92 \mathrm{a}$ & $4.88 \mathrm{a}$ & $4.90 \mathrm{a}$ & $2.34 \mathrm{a}$ & $2.87 \mathrm{a}$ & $2.61 \mathrm{a}$ \\
\hline F. test & $* *$ & $* *$ & $* *$ & $* *$ & $* *$ & $* *$ & $* *$ & $* *$ & $* *$ \\
\hline \multicolumn{10}{|c|}{ Phosphorus level (kg. $\mathrm{P}_{2} \mathrm{O}_{5} /$ fad.) $\mathrm{P}$} \\
\hline $\mathbf{0}$ & $18.30 \mathrm{c}$ & $19.96 \mathrm{c}$ & $19.13 \mathrm{c}$ & $4.02 \mathrm{~b}$ & $4.39 \mathrm{~b}$ & $4.21 \mathrm{~b}$ & $1.80 \mathrm{c}$ & $2.29 \mathrm{c}$ & $2.04 \mathrm{c}$ \\
\hline 15.5 & $19.13 \mathrm{~b}$ & $21.53 \mathrm{~b}$ & $20.33 \mathrm{~b}$ & $4.35 \mathrm{a}$ & $4.51 \mathrm{a}$ & $4.43 \mathrm{a}$ & $2.02 \mathrm{~b}$ & $2.56 \mathrm{~b}$ & $2.29 \mathrm{~b}$ \\
\hline 31 & $20.31 \mathrm{a}$ & $23.46 \mathrm{a}$ & $21.88 \mathrm{a}$ & $4.42 \mathrm{a}$ & $4.61 \mathrm{a}$ & $4.52 \mathrm{a}$ & $2.23 \mathrm{a}$ & $2.74 \mathrm{a}$ & $2.49 \mathrm{a}$ \\
\hline F. test & $* *$ & $* *$ & $* *$ & $* *$ & $*$ & $* *$ & $* *$ & $* *$ & $* *$ \\
\hline \multicolumn{10}{|l|}{ Interactions } \\
\hline $\mathbf{H} \times \mathbf{N}$ & $* *$ & NS & $* *$ & NS & NS & NS & NS & NS & $* *$ \\
\hline $\mathbf{H} \times \mathbf{P}$ & NS & NS & NS & $* *$ & NS & NS & $* *$ & $* *$ & $* *$ \\
\hline $\mathbf{N} \times \mathbf{P}$ & NS & $* *$ & $* *$ & NS & $* *$ & $* *$ & $* *$ & $*$ & $* *$ \\
\hline
\end{tabular}

*,** indicate significant at 0.05 and 0.01 levels of probability, respectively. $\mathrm{NS}=$ Not significant.

\section{Nitrogen level effect}

The results in Table 4 shows that applying $\mathrm{N}$ fertilizer up to $135 \mathrm{~kg} \mathrm{~N} /$ fad., reflected significant effects on maize ear length, ear diameter and stem diameter according to the combined analysis, but $45 \mathrm{~kg} \mathrm{~N} / \mathrm{fad}$., was quite enough to produce the highest averages of ear diameter and stem diameter in the second season. Based on the combined data, adding 45, 90 and $135 \mathrm{~kg} \mathrm{~N} /$ fad., increased ear length by $26.45,34.04$ and $47.58 \%$ and ear diameter increased by $16.57,26.90$ and $33.15 \%$, respectively. These results are in accordance with Vania et al. (2010), Soliman and Gharib (2011), El-Moursy (2013), Seadh et al. (2015) and Matusso and Materusse (2016).

\section{Phosphors level effect}

Results in Table 4 shows that increasing $\mathrm{P}$ level from zero to 15.5 and up to $31 \mathrm{~kg}_{2} \mathrm{O}_{5} /$ fad., caused significant increase in ear length and stem diameter. However addition of the first $\mathrm{P}$ increment $\left(15.5 \quad \mathrm{~kg} \quad \mathrm{P}_{2} \mathrm{O}_{5} /\right.$ fad. $)$, reflected significant increase in ear diameter, whereas the second $\mathrm{P}$ increment failed to add further significant increase. This trend was true in both seasons and their combined. Basing on the combined data, increasing $\mathrm{P}$ levels up to 15.5 and $31 \mathrm{~kg} \mathrm{P}_{2} \mathrm{O}_{5} /$ fad., increased ear length by 6.27 and $14.38 \%$ and stem diameter by 12.25 and $22.06 \%$, respectively compared with the control. These results were in agreement with those of Olusegun (2015).

\section{Interaction effect}

In general, each $\mathrm{N}$ increment was effective to increase significantly ear length for SC. 128 hybrids, however the second $\mathrm{N}$ increment failed to increase ear length of maize hybrid SC 176. Maize hybrids SC. 128 had the higher ear length 
under the different $\mathrm{N}$ level. This was observed also in stem diameter, but maize stem diameter of SC. 128 responded up to $135 \mathrm{~kg} \mathrm{~N} /$ fad., while stem diameter of SC. 176 responded only to 90 $\mathrm{kg} \mathrm{N} /$ fad. (Table 4-a).

It is evident from Table 4-b that each $\mathrm{P}$ increment was effective to increase significantly stem diameter of SC. 176, but stem diameter of SC. 128 responded to $15.5 \mathrm{~kg} \mathrm{P}_{2} \mathrm{O}_{5} /$ fad., only while the second $\mathrm{P}$ increment failed to increase that diameter.

Generally, maize ear length was responded to phosphors fertilizer more when plants were fertilized by $45 \mathrm{~kg} \mathrm{~N} / \mathrm{fad}$. But at both without addition of $\mathrm{N}$ and $90 \mathrm{~kg} \mathrm{~N} / \mathrm{fad}$., ear length was only increased under $\mathrm{P}$ level of $15.5 \mathrm{~kg}$ $\mathrm{P}_{2} \mathrm{O}_{5} /$ fad., when maize was fertilized by $135 \mathrm{~kg}$ $\mathrm{N} /$ fad., the results indicated that ear length consistently increased due to any $\mathrm{P}$ fertilizer increase. Under the $\mathrm{P}$ levels, ear length was affected by $\mathrm{N}$ fertilizer up to $90 \mathrm{~kg} \mathrm{~N} /$ fad., further increase in $\mathrm{N}$ i.e. 135 did not effect ear length (Table 4-b).

Ear diameter of maize was affected by the second increment of $\mathrm{P}$ under $\mathrm{N}$ level of $135 \mathrm{~kg} /$ fad., whereas under control and $90 \mathrm{~kg} \mathrm{~N} / \mathrm{fad}$., this increment failed to increase ear diameter. Under unfertilized plants with $\mathrm{P}$ or adding 15.5 $\mathrm{kg} \mathrm{P}_{2} \mathrm{O}_{5} /$ fad., ear diameter was affected by $\mathrm{N}$ levels up to $45 \mathrm{~kg} \mathrm{~N} / \mathrm{fad}$., when maize fertilized by $31 \mathrm{~kg} \mathrm{P}_{2} \mathrm{O}_{5} /$ fad., that diameter responded only to $90 \mathrm{~kg} \mathrm{~N} / \mathrm{fad}$.

The results indicated that in general, for the two low $\mathrm{N}$ levels i.e. checks and $45 \mathrm{~kg} \mathrm{~N} /$ fad., any increase in phosphorus was followed by a significant increase in stem diameter while under $90 \mathrm{~kg} \mathrm{~N} / \mathrm{fad}$., stem diameter significantly increased by $\mathrm{P}$ application up to $15.5 \mathrm{~kg}$ $\mathrm{P}_{2} \mathrm{O}_{5} /$ fad., but under $135 \mathrm{~kg} \mathrm{~N} /$ fad., the response observed was with $31 \mathrm{~kg} \mathrm{P}_{2} \mathrm{O}_{5} /$ fad. Under either without additional $\mathrm{P}$ or adding $31 \mathrm{~kg}_{2} \mathrm{O}_{5} / \mathrm{fad}$., stem diameter significantly increased by $\mathrm{N}$ application up to $135 \mathrm{~kg} \mathrm{~N} /$ fad., while under $15.5 \mathrm{~kg} \mathrm{P}_{2} \mathrm{O}_{5} /$ fad., that diameter responsed up to $45 \mathrm{~kg} \mathrm{~N} / \mathrm{fad}$.

\section{Number of Rows/Ear, Number of Grains/ Row and Hundred Grain Weight}

Table 5 shows number of rows/ear and number of grains/row as well as hundred grain weight of two maize hybrids as affected by $\mathrm{N}$ and $\mathrm{P}$ levels in the two seasons and their combined.

\section{Maize hybrid differences}

It is seen in Table 5 that the two maize hybrids varied significantly regarding each of number of grains/row and hundred grain weight while number of rows/ear was insignificantly affected. SC. 128 had more number of grains/ row (44.42) than SC. 176 (40.07) and also gave heavier hundred grain weight (37.89) than SC. 176 (33.76). The two hybrids had almost similar number of rows/ear. This was true in the two seasons and was ascertained by the combined analysis.

Significant hybrid differences were reported in the literature regarding number of grains/row Attia et al. (2009), Sharifi et al. (2009), Mukhtar et al. (2012) and Hejazi and Soleymani (2014). and hundred grain weight Attia et al. (2009), Abdou et al. (2012), Modhej et al. (2014) and Sorkhi and Fateh (2014).

\section{Nitrogen level effect}

Each $\mathrm{N}$ increment up to the highest $\mathrm{N}$ level under study (135 kg N/fad.), was accompanied by a significant increase in each of number of rows/row, number of grains/row and hundred grain weight in the two seasons and their combined with the exception of the second season of number of rows/ear (Table 5) Based on the combined data adding 45, 90 and $135 \mathrm{~kg}$ $\mathrm{N} /$ fad., increased number of grains/row by $22.13,36.27$ and $46.46 \%$ and hundred grain weight by $32.15,39.32$ and $47.43 \%$, respectively.

Similar increases were observed in chlorophyll content (Table 1), plant height (Table 2) and LAI (Table 3). These results clearly indicated that the increase of ear diameter due to the increase of $\mathrm{N}$ levels was significantly effected on number of rows/ear and these $\mathrm{N}$ increments increased each of ear length (Table 4) which resulted in a significant increase in the number of grains/row and finally the number of grains/ear. These results followed the same patterns of the most yield attributes former discussed indicating the promotion effect of $\mathrm{N}$ on vegetative growth which inturn favored metabolic processes and increased growth and yield attributes, of corn. In the literature, several authors found that addition of $\mathrm{N}$ was effective to increase each of number of rows/ear and number 
Table 4-a. Effect of interaction between maize hybrids and nitrogen fertilizer levels on ear length $(\mathrm{cm})$ and stem diameter $(\mathrm{cm})$ (combined)

\begin{tabular}{lcccccccc}
\hline $\begin{array}{l}\text { Maize hybrid } \\
\text { H) }\end{array}$ & \multicolumn{9}{c}{ Ear length (cm) } \\
\cline { 2 - 10 } & \multicolumn{1}{c}{$\mathbf{0}$} & $\mathbf{4 5}$ & $\mathbf{9 0}$ & $\mathbf{1 3 5}$ & $\mathbf{0}$ & $\mathbf{4 5}$ & $\mathbf{9 0}$ & $\mathbf{1 3 5}$ \\
\cline { 2 - 10 } & $\mathrm{D}$ & $\mathrm{C}$ & $\mathrm{B}$ & $\mathrm{A}$ & $\mathrm{C}$ & $\mathrm{B}$ & $\mathrm{B}$ & $\mathrm{A}$ \\
& $16.82 \mathrm{a}$ & $21.47 \mathrm{a}$ & $22.93 \mathrm{a}$ & $25.55 \mathrm{a}$ & $1.79 \mathrm{a}$ & $2.77 \mathrm{a}$ & $2.76 \mathrm{a}$ & $2.91 \mathrm{a}$ \\
SC 128 & $\mathrm{C}$ & $\mathrm{B}$ & $\mathrm{B}$ & $\mathrm{A}$ & $\mathrm{C}$ & $\mathrm{B}$ & $\mathrm{AB}$ & $\mathrm{A}$ \\
& $15.38 \mathrm{~b}$ & $19.24 \mathrm{~b}$ & $20.23 \mathrm{~b}$ & $21.96 \mathrm{~b}$ & $1.33 \mathrm{~b}$ & $2.09 \mathrm{~b}$ & $2.22 \mathrm{~b}$ & $2.30 \mathrm{~b}$ \\
\hline
\end{tabular}

Table 4-b. Effect of interaction between maize hybrids and phosphorus fertilizer levels on stem diameter (cm) (combined)

\begin{tabular}{lccc}
\hline $\begin{array}{l}\text { Maize hybrid } \\
\text { (H) }\end{array}$ & \multicolumn{3}{c}{ Stem diameter $(\mathrm{cm})$} \\
\cline { 2 - 4 } & \multicolumn{3}{c}{ P level $\left(\mathrm{kg} \mathrm{P}_{2} \mathrm{O}_{5} /\right.$ fad.) } \\
\cline { 2 - 4 } & $\mathrm{O}$ & 15.5 & 31 \\
\hline \multirow{3}{*}{ SC 128} & $\mathrm{~B}$ & $\mathrm{~A}$ & $\mathrm{~A}$ \\
& $2.29 \mathrm{a}$ & $2.64 \mathrm{a}$ & $2.74 \mathrm{a}$ \\
SC 176 & $\mathrm{C}$ & $\mathrm{B}$ & $\mathrm{A}$ \\
\hline
\end{tabular}

Table 4-c. Effect of interaction between nitrogen fertilizer levels and phosphorus fertilizer levels on ear length $(\mathrm{cm})$, ear diameter $(\mathrm{cm})$ and stem diameter $(\mathrm{cm})$ (combined)

\begin{tabular}{|c|c|c|c|c|c|c|c|c|c|}
\hline \multirow{3}{*}{$\begin{array}{l}\text { N level } \\
\text { (kg N/fad.) }\end{array}$} & \multicolumn{3}{|c|}{ Ear length (cm) } & \multicolumn{3}{|c|}{ Ear diameter (cm) } & \multicolumn{3}{|c|}{ Stem diameter $(\mathrm{cm})$} \\
\hline & \multicolumn{9}{|c|}{$P$ level (kg $\mathrm{P}_{2} \mathrm{O}_{5} /$ fad.) } \\
\hline & $\mathbf{0}$ & $\mathbf{1 5 . 5}$ & 31 & $\mathbf{0}$ & $\mathbf{1 5 . 5}$ & 31 & $\mathbf{0}$ & $\mathbf{1 5 . 5}$ & 31 \\
\hline \multirow{2}{*}{ 0 } & B & $\mathrm{A}$ & A & A & $\mathrm{A}$ & A & $\mathrm{C}$ & B & A \\
\hline & $19.41 \mathrm{~b}$ & $20.08 \mathrm{~b}$ & $21.07 \mathrm{~b}$ & $3.53 \mathrm{~b}$ & $3.82 \mathrm{~b}$ & $3.68 \mathrm{c}$ & $1.20 \mathrm{c}$ & $1.59 \mathrm{~b}$ & $1.89 \mathrm{c}$ \\
\hline \multirow{2}{*}{45} & $\mathrm{~B}$ & $\mathrm{~B}$ & $\mathrm{~A}$ & $\mathrm{~A}$ & $\mathrm{~A}$ & $\mathrm{~B}$ & $\mathrm{C}$ & B & $\mathrm{A}$ \\
\hline & $17.19 \mathrm{c}$ & $18.18 \mathrm{c}$ & $19.56 \mathrm{c}$ & $4.37 \mathrm{a}$ & $4.36 \mathrm{a}$ & $4.13 \mathrm{~b}$ & $2.23 \mathrm{~b}$ & $2.46 \mathrm{a}$ & $2.62 \mathrm{~b}$ \\
\hline \multirow{2}{*}{90} & B & A & A & B & A & A & B & A & A \\
\hline & $21.56 \mathrm{a}$ & $23.35 \mathrm{a}$ & $24.68 \mathrm{a}$ & $4.27 \mathrm{ab}$ & $4.79 \mathrm{a}$ & $4.96 \mathrm{a}$ & $2.30 \mathrm{~b}$ & $2.56 \mathrm{a}$ & $2.60 \mathrm{~b}$ \\
\hline \multirow{2}{*}{135} & $\mathrm{C}$ & $\mathrm{B}$ & $\mathrm{A}$ & $\mathrm{B}$ & $\mathrm{B}$ & $\mathrm{A}$ & $\mathrm{B}$ & $\mathrm{B}$ & $\mathrm{A}$ \\
\hline & $18.35 \mathrm{~b}$ & $19.71 \mathrm{~b}$ & $22.24 \mathrm{~b}$ & $4.66 \mathrm{a}$ & $4.76 \mathrm{a}$ & $5.29 \mathrm{a}$ & $2.45 \mathrm{a}$ & $2.538 \mathrm{a}$ & $2.83 \mathrm{a}$ \\
\hline
\end{tabular}


Table 5. Number of rows/ear, number of grains/row and 100-grain weight (g) of the two maize hybrids as affected by nitrogen fertilizer levels, phosphorus fertilizer levels and their interactions in 2014, 2015 seasons and their combined data.

\begin{tabular}{|c|c|c|c|c|c|c|c|c|c|}
\hline \multirow{2}{*}{$\begin{array}{l}\text { Main effects and } \\
\text { interactions } \\
\end{array}$} & \multicolumn{3}{|c|}{ Number of rows/ear } & \multicolumn{3}{|c|}{ Number of grains/row } & \multicolumn{3}{|c|}{ 100-grain weight (g) } \\
\hline & 2014 & 2015 & Comb. & 2014 & 2015 & Comb. & 2014 & 2015 & Comb. \\
\hline \multicolumn{10}{|l|}{ Maize Hybrid (H) } \\
\hline SC 128 & 15.26 & 14.95 & 15.11 & $43.10 \mathrm{a}$ & $45.73 \mathrm{a}$ & $44.42 \mathrm{a}$ & $37.02 \mathrm{a}$ & $38.77 \mathrm{a}$ & $37.89 \mathrm{a}$ \\
\hline SC 176 & 14.85 & 15.00 & 14.92 & $38.64 \mathrm{~b}$ & $41.50 \mathrm{~b}$ & $40.07 \mathrm{~b}$ & $32.77 \mathrm{~b}$ & $34.76 \mathrm{~b}$ & $33.76 \mathrm{~b}$ \\
\hline F. test & NS & NS & NS & ** & ** & $* *$ & ** & ** & ** \\
\hline
\end{tabular}

Nitrogen level (kg N/fad.) $\mathbf{N}$

$\begin{array}{llllllllll}\mathbf{0} & 13.46 \mathrm{~d} & 14.10 \mathrm{~b} & 13.78 \mathrm{~d} & 32.97 \mathrm{~d} & 33.96 \mathrm{~d} & 33.47 \mathrm{~d} & 28.26 \mathrm{~d} & 26.98 \mathrm{~d} & 27.62 \mathrm{~d} \\ \mathbf{4 5} & 14.42 \mathrm{c} & 14.67 \mathrm{ab} & 14.54 \mathrm{c} & 39.06 \mathrm{c} & 42.71 \mathrm{c} & 40.88 \mathrm{c} & 35.12 \mathrm{c} & 37.88 \mathrm{c} & 36.50 \mathrm{c} \\ \mathbf{9 0} & 15.59 \mathrm{~b} & 15.22 \mathrm{a} & 15.41 \mathrm{~b} & 43.68 \mathrm{~b} & 47.54 \mathrm{~b} & 45.61 \mathrm{~b} & 36.99 \mathrm{~b} & 39.97 \mathrm{~b} & 38.48 \mathrm{~b} \\ \mathbf{1 3 5} & 16.74 \mathrm{a} & 15.92 \mathrm{a} & 16.33 \mathrm{a} & 47.78 \mathrm{a} & 50.26 \mathrm{a} & 49.02 \mathrm{a} & 39.21 \mathrm{a} & 42.23 \mathrm{a} & 40.72 \mathrm{a}\end{array}$

F. test

Phosphorus level (kg. $\mathbf{P}_{2} \mathrm{O}_{5} /$ fad.) $\mathbf{P}$

$\mathbf{0}$

15.5

31

F. test

Interactions

$\mathbf{H} \times \mathbf{N}$
$\mathbf{H} \times \mathbf{P}$
$\mathbf{N} \times \mathbf{P}$
14.41 c 14.68 b 14.54 c 38.63 c 41.38 c 40.00 c 32.93 c 34.00 c 33.46 c

$\begin{array}{lllllllllllllll}15.06 & b & 14.82 b & 14.94 b & 40.46 b & 43.09 b & 41.77 b & 35.23 b & 37.10 b & 36.16 b\end{array}$

15.69 a 15.43 a 15.56 a 43.53 a 46.38 a 44.95 a 36.53 a 39.19 a 37.86 a

*** indicate significant at 0.05 and 0.01 levels of probability, respectively. $\mathrm{NS}=$ Not significant.

of grains/row and hundred grain weight such as Sharifi and Taghizadeh (2009), Kandil (2013), Seadh et al. (2015) and Matusso and Materusse (2016).

\section{Phosphors level effect}

It is evident from Table 5 that each $\mathrm{P}$ increment up to $31 \mathrm{~kg} \mathrm{P}_{2} \mathrm{O}_{5} /$ fad., produced significant increase in each of row number/ear, grain number/row and hundred grain weight in both seasons and their combined. Also, the addition of $31 \mathrm{~kg} \mathrm{P}_{2} \mathrm{O}_{5} /$ fad., was followed by a significant increase in row number/ear, but the first $\mathrm{P}$ increment failed to add a significant increase in this number in the first season. Basing on the combined data, increasing $\mathrm{P}$ level up to $31 \mathrm{~kg} \mathrm{P}_{2} \mathrm{O}_{5} /$ fad., increased row number/ear by 2.75 and $7.02 \%$ and grain number/row by 4.42 and $12.38 \%$ as well as hundred grain weight by 8.07 and $13.15 \%$, respectively compared with the control. Similar results were reported by Khan et al. (2005), Ahmad et al. (2007) and Mukhtar et al. (2011).

\section{Interaction effect}

The two maize hybrids used had the same number of rows/ear under each of check, 45 and $135 \mathrm{~kg} \mathrm{~N} /$ fad. It seemed that SC. 128 hybrid had higher No. of rows/ear than SC. 176 when $90 \mathrm{~kg}$ $\mathrm{N} /$ fad., was applied. Regarding to SC. 128, any increase in nitrogen fertilizer up to $90 \mathrm{~kg} \mathrm{~N} /$ fad., caused a significant increase in number of rows/ ear, (Table 5-a). 
It seemed that SC. 128 hybrid always had higher number of grains/row and hundred grain weight than SC. 176 hybrid under the different $\mathrm{N}$ level. For the two maize hybrids used, any increase in nitrogen fertilizer caused a significant increase in both number of grains/ row and hundred grain weight (Table 5-a).

The maize hybrids used had the same number of rows/ear under the check P treatment. It seemed that SC. 128 hybrid always had higher number of rows/ear than SC. 176 when either 15.5 or $31 \mathrm{~kg} \mathrm{P}_{2} \mathrm{O}_{5} /$ fad., was applied. For SC. 128 hybrid any increase in phosphors fertilizer caused a significant increase in number of rows/ ear, however, this was always observed for the second P increment in SC. 176 case Table (5-b).

Under growing maize without applying $\mathrm{P}$ fertilizer or $15.5 \mathrm{~kg} \mathrm{P}_{2} \mathrm{O}_{5} /$ fad., the number of rows/ear responded to nitrogen fertilizer up to $135 \mathrm{~kg} \mathrm{~N} /$ fad.. In general, number of rows/ear was affected by the first and second $\mathrm{P}$ increment under both 90 and $135 \mathrm{~kg} \mathrm{~N} /$ fad., but it only increased due to applying $90 \mathrm{~kg} \mathrm{~N} /$ fad., under 31 $\mathrm{kg} \mathrm{P}_{2} \mathrm{O}_{5} / \mathrm{fad}$. Thus, under $45 \mathrm{~kg} \mathrm{~N} / \mathrm{fad}$., there was no significant different between the three $\mathrm{P}$ levels, where the first $P$ increment increased that number only when maize unfertilized by nitrogen.

Under any phosphorus treatment, increasing nitrogen fertilizer from check to 45 or from 45 to 90 or from 90 to 135 was accompanied with significant increase in number of grains/row. In general, number of grains/row was affected by the first and second $\mathrm{P}$ increment for the different $\mathrm{N}$ levels. Also the same trend was observed in hundred grain weight, but under $45 \mathrm{~kg} \mathrm{~N} /$ fad., that weight was affected only by the first $\mathrm{P}$ increment (Table 5-c).

\section{Grain, Stover and Biological Yields/Plant}

Table 6 shows grain, stover and biological yields/plant of the two maize hybrids as affected by $\mathrm{N}$ and $\mathrm{P}$ levels and their interaction in both seasons and their combined.

\section{Maize hybrid differences}

Single cross 128 maize hybrid surpassed noticeably SC. 176 one for grain and stover as well as biological yields/plant, since the former had $9.10,23.26$ and $18.15 \%$ more yields than the latter and this was clearly valid in both growing seasons and across their pooled data (Table 6). The increase in grain yield/plant of SC. 128 could be attributed to larger number of grains/row and greater weight of hundred grains if compared with the other tested SC. 176. Cultivar and hybrids differences in yield/plant were reported by other investigators of than Ghanem et al. (2006), Sharifi et al. (2009), Abdou et al. (2012), Golezani and Tajbakhsh (2012), Kandil (2013) and Modhej et al. (2014).

\section{Nitrogen level effect}

Each $\mathrm{N}$ increment produced a significant increase in each of grain yield/plant, stover yield/ plant and biological yield/plant. This was valid in the two seasons and their combined. According to the combined analysis, the percentage increase due to addition of $135 \mathrm{~kg}$ $\mathrm{N} /$ fad., were $186.6,57.25$ and $90.52 \%$ for grain yield/plant, stover yield/plant and biological yield/plant. Similar effects were observed in plant height (Table 2), ear length and diameter (Table 4), row number/ear and grain number/ row as well as hundred grain weight (Table 5). It is worthy noticed that, $\mathrm{N}$ added to maize plants enhanced photosynthesis and photosynthetic partitioning to the grains as a results of raising enzymatic activity and other biological processes inside maize plant. These results are in accordance with those given by Azeez et al. (2006), El-Moursy (2013), Hejazi and Soleymani (2014), Seadh et al. (2015) and Matusso and Materusse (2016).

\section{Phosphors level effect}

Results in Table 6 shows that increasing $\mathrm{P}$ levels from zero to 15.5 and $31 \mathrm{~kg} \mathrm{P}_{2} \mathrm{O}_{5} / \mathrm{fad}$., caused significant increase in grain yield/plant and stover yield/plant as well as biological yield/ plant. This trend was true in both seasons and their combined. Basing on the combined data increasing P levels up to 15.5 and $31 \mathrm{~kg} \mathrm{P}_{2} \mathrm{O}_{5} /$ fad., increased grain yield/plant by 33.09 and $63.22 \%$ and stover yield/plant by 6.23 and $11.63 \%$ as well as biological yield by 14.24 and $27.03 \%$, respectively compared with the control. These results are in consistent with those reported on other yield attributes namely plant height, ear leaf area, leaf area index, relative photosynthetic potential for grain, ear length, number of rows/ear, number of grains/ row and hundred grain weight (Tables 2, 3, 4 and 5). 
Zagazig J. Agric. Res., Vol. 44 No. (1) 2017

Table 5-a. Effect of interaction between maize hybrids and nitrogen fertilizer levels on number of rows/ear, number of grains/ row and 100-grain weight (g) (combined)

\begin{tabular}{|c|c|c|c|c|c|c|c|c|c|c|c|c|}
\hline \multirow{3}{*}{$\begin{array}{l}\text { Maize hybrid } \\
\text { (H) }\end{array}$} & \multicolumn{4}{|c|}{ Number of rows/ ear } & \multicolumn{4}{|c|}{ Number of grains/row } & \multicolumn{4}{|c|}{ 100-grain weight (g) } \\
\hline & \multicolumn{12}{|c|}{ N level (kg N/fad.) } \\
\hline & $\mathbf{0}$ & 45 & 90 & 135 & $\mathbf{0}$ & 45 & 90 & 135 & $\mathbf{0}$ & 45 & 90 & 135 \\
\hline & $\mathrm{C}$ & B & A & A & D & $\mathrm{C}$ & B & A & D & $\mathrm{C}$ & B & A \\
\hline
\end{tabular}

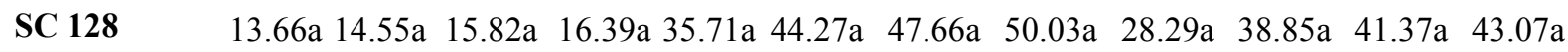

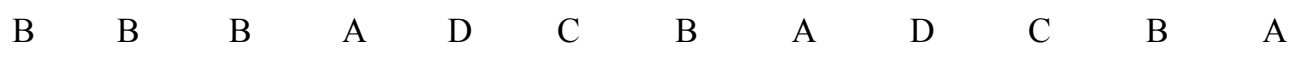

SC 176

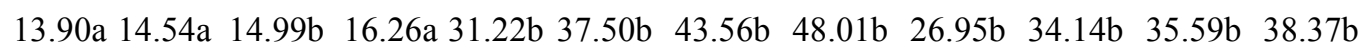

Table 5-b. Effect of interaction between maize hybrids and phosphorus fertilization levels on number of rows/ear (combined)

\begin{tabular}{lccc}
\hline Maize hybrid & \multicolumn{3}{c}{ Number of rows/ ear } \\
\cline { 2 - 4 } & \multicolumn{3}{c}{ P level (kg $\mathbf{P}_{2} \mathbf{O}_{\mathbf{5}} /$ fad.) } \\
\cline { 2 - 4 } & $\mathbf{0}$ & $\mathbf{1 5 . 5}$ & $\mathbf{3 1}$ \\
\hline \multirow{2}{*}{ SC 128 } & $\mathrm{C}$ & $\mathrm{B}$ & $\mathrm{A}$ \\
& $14.40 \mathrm{a}$ & $15.07 \mathrm{a}$ & $15.85 \mathrm{a}$ \\
SC 176 & $\mathrm{B}$ & $\mathrm{B}$ & $\mathrm{A}$ \\
\hline
\end{tabular}

Table 5-c. Effect of interaction between nitrogen fertilizer levels and phosphorus fertilizer levels on number of rows/ear, number of grains/row and 100-grain weight (g) (combined)

\begin{tabular}{|c|c|c|c|c|c|c|c|c|c|}
\hline \multirow{3}{*}{$\begin{array}{l}\text { N level } \\
\text { (kg N/fad.) }\end{array}$} & \multicolumn{3}{|c|}{ Number of rows/ ear } & \multicolumn{3}{|c|}{ Number of grains/row } & \multicolumn{3}{|c|}{100 - grain weight $(\mathrm{g})$} \\
\hline & \multicolumn{9}{|c|}{ P level (kg $\mathrm{P}_{2} \mathrm{O}_{5} /$ fad.) } \\
\hline & 0 & 15.5 & 31 & 0 & 15.5 & 31 & 0 & 15.5 & 31 \\
\hline \multirow{3}{*}{$\mathbf{0}$} & $\mathrm{B}$ & $\mathrm{AB}$ & $\mathrm{A}$ & $\mathrm{C}$ & B & $\mathrm{A}$ & $\mathrm{C}$ & $\mathrm{B}$ & $\mathrm{A}$ \\
\hline & $13.42 \mathrm{c}$ & $13.72 \mathrm{c}$ & $14.22 \mathrm{~b}$ & $31.81 \mathrm{~d}$ & $33.52 \mathrm{~d}$ & $35.07 \mathrm{~d}$ & $22.56 \mathrm{~d}$ & $28.61 \mathrm{~d}$ & $31.69 \mathrm{~d}$ \\
\hline & A & A & $\mathrm{A}$ & $\mathrm{C}$ & B & A & B & A & A \\
\hline \multirow[t]{2}{*}{45} & $14.37 \mathrm{~b}$ & $14.45 \mathrm{c}$ & $14.83 \mathrm{~b}$ & $38.41 \mathrm{c}$ & $41.28 \mathrm{c}$ & $42.97 \mathrm{c}$ & $35.18 \mathrm{c}$ & $36.83 \mathrm{c}$ & $37.48 \mathrm{c}$ \\
\hline & C & $\mathrm{B}$ & A & $\mathrm{C}$ & $\mathrm{B}$ & A & $\mathrm{C}$ & $\mathrm{B}$ & A \\
\hline \multirow[t]{2}{*}{90} & $14.50 \mathrm{~b}$ & $15.41 \mathrm{~b}$ & $16.31 \mathrm{a}$ & $42.45 \mathrm{~b}$ & $43.78 \mathrm{~b}$ & $50.59 \mathrm{~b}$ & $37.32 \mathrm{~b}$ & $38.29 \mathrm{~b}$ & $39.83 \mathrm{~b}$ \\
\hline & $\mathrm{C}$ & B & A & $\mathrm{C}$ & $\mathrm{B}$ & A & $\mathrm{C}$ & B & A \\
\hline 135 & $15.90 \mathrm{a}$ & $16.18 \mathrm{a}$ & $16.90 \mathrm{a}$ & $47.35 \mathrm{a}$ & $48.52 \mathrm{a}$ & $51.188 \mathrm{a}$ & $38.78 \mathrm{a}$ & $40.93 \mathrm{a}$ & $42.44 \mathrm{a}$ \\
\hline
\end{tabular}


Table 6. Grain yield (g./plant), stover yield (g./plant) and biological yield (g./plant) of the two maize hybrids as affected by nitrogen, phosphorus fertilizer levels and their interactions in 2014, 2015 seasons and their combined data

\begin{tabular}{|c|c|c|c|c|c|c|c|c|c|}
\hline \multirow{2}{*}{$\begin{array}{l}\text { Main effects and } \\
\text { interactions }\end{array}$} & \multicolumn{3}{|c|}{ Grain yield (g./plant) } & \multicolumn{3}{|c|}{ Stover yield (g./plant) } & \multicolumn{3}{|c|}{ Biological yield (g./plant) } \\
\hline & 2014 & 2015 & Comb. & 2014 & 2015 & Comb. & 2014 & 2015 & Comb. \\
\hline \multicolumn{10}{|l|}{ Maize hybrid (H) } \\
\hline SC 128 & $136.21 \mathrm{a}$ & $151.08 \mathrm{a}$ & $143.65 \mathrm{a}$ & $285.25 \mathrm{a}$ & $288.16 \mathrm{a}$ & $286.70 \mathrm{a}$ & $421.46 \mathrm{a}$ & $439.24 a$ & $430.35 \mathrm{a}$ \\
\hline SC 176 & $125.08 \mathrm{~b}$ & $138.24 b$ & $131.66 \mathrm{~b}$ & $229.09 b$ & $236.10 \mathrm{~b}$ & $232.59 b$ & $354.17 \mathrm{~b}$ & $374.33 b$ & $364.25 b$ \\
\hline F. test & $* *$ & $* *$ & $* *$ & $* *$ & $* *$ & $* *$ & $* *$ & $* *$ & $* *$ \\
\hline \multicolumn{10}{|c|}{ Nitrogen level (kg N/fad.) N } \\
\hline $\mathbf{0}$ & $65.97 \mathrm{~d}$ & $69.69 \mathrm{~d}$ & $67.45 d$ & $197.88 d$ & $191.94 d$ & $194.91 d$ & $263.08 \mathrm{~d}$ & $261.63 d$ & $262.36 \mathrm{~d}$ \\
\hline 45 & $120.65 c$ & $135.66 \mathrm{c}$ & $128.16 \mathrm{c}$ & $251.69 \mathrm{c}$ & $257.60 \mathrm{c}$ & $254.64 \mathrm{c}$ & $372.34 c$ & $393.26 \mathrm{c}$ & $382.80 \mathrm{c}$ \\
\hline 90 & $151.49 b$ & $171.78 b$ & $161.63 \mathrm{~b}$ & $275.40 \mathrm{~b}$ & $289.70 \mathrm{~b}$ & $282.55 b$ & $426.88 b$ & $461.48 b$ & $444.18 b$ \\
\hline 135 & $185.24 \mathrm{a}$ & $201.50 \mathrm{a}$ & $193.37 \mathrm{a}$ & $303.71 \mathrm{a}$ & $309.28 \mathrm{a}$ & $306.50 \mathrm{a}$ & $488.95 \mathrm{a}$ & $510.78 \mathrm{a}$ & $499.87 \mathrm{a}$ \\
\hline F. test & $* *$ & $* *$ & $* *$ & $* *$ & $* *$ & $* *$ & $* *$ & $* *$ & $* *$ \\
\hline \multicolumn{10}{|c|}{ Phosphorus level (kg. $\mathrm{P}_{2} \mathrm{O}_{5} /$ fad.) $\mathrm{P}$} \\
\hline $\mathbf{0}$ & $98.59 \mathrm{c}$ & $109.80 \mathrm{c}$ & $104.20 \mathrm{c}$ & $239.20 c$ & $250.91 \mathrm{c}$ & $245.06 \mathrm{c}$ & $337.79 c$ & $360.71 \mathrm{c}$ & $349.25 \mathrm{c}$ \\
\hline 15.5 & $130.23 b$ & $147.12 b$ & $138.68 b$ & $261.22 b$ & $259.42 b$ & $260.32 b$ & $391.45 b$ & $406.54 b$ & $398.99 b$ \\
\hline 31 & $163.11 \mathrm{a}$ & $177.06 \mathrm{a}$ & $170.08 \mathrm{a}$ & $271.09 \mathrm{a}$ & $276.06 \mathrm{a}$ & $273.57 \mathrm{a}$ & $434.20 \mathrm{a}$ & $453.11 \mathrm{a}$ & $443.66 \mathrm{a}$ \\
\hline F. test & $* *$ & $* *$ & $* *$ & $* *$ & $* *$ & $* *$ & $* *$ & $* *$ & $* *$ \\
\hline \multicolumn{10}{|l|}{ Interactions } \\
\hline $\mathbf{H} \times \mathbf{N}$ & $*$ & $*$ & $* *$ & $* *$ & $* *$ & $* *$ & $* *$ & $* *$ & $* *$ \\
\hline $\mathbf{H} \times \mathbf{P}$ & $* *$ & NS & $* *$ & NS & NS & NS & NS & NS & NS \\
\hline $\mathbf{N} \times \mathbf{P}$ & $* *$ & $* *$ & $* *$ & $* *$ & $* *$ & $* *$ & $* *$ & $* *$ & $* *$ \\
\hline
\end{tabular}

In this regard Khan et al. (2005), Hussein (2009) and Popoola et al. (2015) obtained similar results.

\section{Interaction effect}

In general, each $\mathrm{N}$ increment was effective to increase both grain and biological yields/plant for the two maize hybrids i.e. SC. 128 and SC.176. It seemed that SC. 128 hybrid always had higher yield/plant than SC.176 under the different $\mathrm{N}$ levels, but the maize hybrids had the same grain yield under check $\mathrm{N}$ treatment.

For SC. 176 hybrid the second $\mathrm{N}$ increment failed to increase stover yield/plant, but any $\mathrm{N}$ increment increased stover yields/plant for SC. 128. In general SC. 128 hybrid always had higher stover and biological yields/plant than SC. 176 under the different $\mathrm{N}$ levels (Table 6-a). It is evident from Table 6-b that each $P$ increment was effective to increase grain yield/ plant of the two maize hybrids i.e. SC. 128 and SC. 176 significantly. SC. 128 hybrid had the higher grain yield/plant under both check $\mathrm{P}$ and $31 \mathrm{~kg} \mathrm{P}_{2} \mathrm{O}_{5} /$ fad., but both SC. 128 and SC. 176 had the same grain yield/plant under $15.5 \mathrm{~kg}$ $\mathrm{P}_{2} \mathrm{O}_{5} /$ fad.

It is evident from Table 6-c that each $\mathrm{N}$ increment produced a significant increase in the grain yield/plant under the two high levels of phosphorus i.e. 15.5 and $31 \mathrm{~kg} \mathrm{P}_{2} \mathrm{O}_{5} /$ fad. Where, no further significant increase could be obtained due to the increase of $\mathrm{N}$ level beyond $90 \mathrm{~kg}$ $\mathrm{N} /$ fad., under the check $\mathrm{P}$ treatment. Also grain yield/plant was affected by the first and second $\mathrm{P}$ increment for each of unfertilized by $\mathrm{N}, 90$ and $135 \mathrm{~kg} \mathrm{~N} / \mathrm{fad}$. But no further significant could be obtained due to the increase of $\mathrm{P}$ level beyond $15.5 \mathrm{~kg} \mathrm{P}_{2} \mathrm{O}_{5} /$ fad., under the $45 \mathrm{~kg}$ $\mathrm{N} /$ fad., treatment. 
Table 6-a. Effect of interaction between maize hybrids and nitrogen fertilizer levels on grain yield (g/plant), stover yield (g/plant) and biological yield (g/plant) (combined)

\begin{tabular}{|c|c|c|c|c|c|c|c|c|c|c|c|c|}
\hline \multirow{3}{*}{$\begin{array}{l}\text { Maize hybrid } \\
\text { (H) }\end{array}$} & \multicolumn{4}{|c|}{ Grain yield (g/plant) } & \multicolumn{4}{|c|}{ Stover yield (g/plant) } & \multicolumn{4}{|c|}{ Biological yield (g/plant) } \\
\hline & \multicolumn{12}{|c|}{ N level (kg N/fad.) } \\
\hline & $\mathbf{0}$ & 45 & 90 & 135 & $\mathbf{0}$ & 45 & 90 & 135 & $\mathbf{0}$ & 45 & 90 & 135 \\
\hline \multirow{3}{*}{ SC 128} & $\mathrm{D}$ & $\mathrm{C}$ & $\mathrm{B}$ & A & $\mathrm{D}$ & $\mathrm{C}$ & $\mathrm{B}$ & $\mathrm{A}$ & $\mathrm{D}$ & $\mathrm{C}$ & $\mathrm{B}$ & A \\
\hline & $66.85 \mathrm{a}$ & 135.89 & 169.21 & 202.63 & 207.4 & 279.02 & $313.54 a$ & 346.82 & 274.29 & 414.91 & 82.7 & $549.44 a$ \\
\hline & $\mathrm{D}$ & $\mathrm{C}$ & $\mathrm{B}$ & A & $\mathrm{C}$ & $\mathrm{B}$ & A & A & $\mathrm{D}$ & $\mathrm{C}$ & B & A \\
\hline SC 176 & \multicolumn{12}{|c|}{ 68.04a 120.42b 154.05b 184.11b 182.38b 230.27b 251.58b 266.18b 250.42b 350.69b 405.61b 450.29b } \\
\hline
\end{tabular}

Table 6-b. Effect of interaction between maize hybrids and phosphorus fertilizer levels on grain yield (g/plant) (combined)

\begin{tabular}{lccc}
\hline Maize hybrid & \multicolumn{3}{c}{ Grain yield (g/plant) } \\
\cline { 2 - 4 } & $\mathbf{3}$ P level (kg $\mathbf{P}_{\mathbf{2}} \mathbf{O}_{\mathbf{5}} /$ fad.) \\
\cline { 2 - 4 } & $\mathbf{0}$ & $\mathbf{1 5 . 5}$ & $\mathbf{3 1}$ \\
\hline & $\mathrm{C}$ & $\mathrm{B}$ & $\mathrm{A}$ \\
SC 128 & $111.27 \mathrm{a}$ & $141.30 \mathrm{a}$ & $178.37 \mathrm{a}$ \\
& $\mathrm{C}$ & $\mathrm{B}$ & $\mathrm{A}$ \\
SC 176 & $97.12 \mathrm{~b}$ & $136.05 \mathrm{a}$ & $161.79 \mathrm{~b}$ \\
\hline
\end{tabular}

Under growing maize without applying $\mathrm{P}$ fertilizer or $31 \mathrm{~kg} \mathrm{P}_{2} \mathrm{O}_{5} /$ fad., stover yield/plant responded to nitrogen fertilizer up to $135 \mathrm{~kg}$ $\mathrm{N} /$ fad., but only increased till $90 \mathrm{~kg} / \mathrm{fad}$., under $15.5 \mathrm{~kg} \mathrm{P}_{2} \mathrm{O}_{5} /$ fad.. In general, each $\mathrm{P}$ increment produced a significant increase in stover yield/ plant under the check $\mathrm{N}$ fertilizer, whereas, no further significant increase could be obtained due to the increase of $\mathrm{P}$ level beyond $15.5 \mathrm{~kg}$ $\mathrm{P}_{2} \mathrm{O}_{5} /$ fad., at both 90 and $135 \mathrm{~kg} \mathrm{~N} /$ fad.. But at $45 \mathrm{~kg} \mathrm{~N} /$ fad., the stover yield did not affected by various $\mathrm{P}$ levels, each $\mathrm{N}$ increment produced a significant increase in the biological/plant. Also biological yield/plant was affected by the first and second $\mathrm{P}$ increment under both 90 and 135 $\mathrm{kg} / \mathrm{fad}$., whereas the last $\mathrm{P}$ increment failed to add a significant increase under both check $\mathrm{N}$ and $45 \mathrm{~kg} \mathrm{~N} /$ fad.

\section{Grain and Biological Yields/fad.}

Grain and biological yields/fad., as affected by the different $\mathrm{N}$ and $\mathrm{P}$ levels and their interactions in the two seasons and their combined is given in Table 7 for two maize hybrids.

\section{Maize hybrid differences}

The two maize hybrids exerted significant variation in both grain and biological yields/fad., where SC. 128 had greater grain and biological yields than the other corresponding SC. 176 one. This inclination was fairly virtual in both growing seasons and affirmed when their data were statistically combined. Basing on the combined data, the grain and biological yields obtained from SC. 128 was about 10.19 and $27.83 \%$ greater than the other tested SC. 176 , respectively. The hybrid differences in such trait could be explained by the fact that the photosynthetic translocate from the source to the sink were great enough to fill all the grains of SC. 128 hybrid or to increase the final grain yield/ fad., relative to SC. 176 one. On the other 
Table 6-c. Effect of interaction between nitrogen fertilizer levels and phosphorus fertilizer levels on grain yield (g/plant), stover yield (g/plant) and biological yield (g/plant) (combined)

\begin{tabular}{|c|c|c|c|c|c|c|c|c|c|}
\hline \multirow{3}{*}{$\begin{array}{l}\text { N level } \\
\text { (kg N/fad.) }\end{array}$} & \multicolumn{3}{|c|}{ Grain yield (g/plant) } & \multirow{2}{*}{\multicolumn{3}{|c|}{$\begin{array}{l}\text { Stover yield (g/plant) } \\
\text { P level (kg } \mathrm{P}_{2} \mathrm{O}_{5} / \text { fad.) }\end{array}$}} & \multicolumn{3}{|c|}{ Biological yield (g/plant) } \\
\hline & & & & & & & & & \\
\hline & $\mathbf{0}$ & 15.5 & 31 & $\mathbf{0}$ & 15.5 & 31 & $\mathbf{0}$ & 15.5 & 31 \\
\hline \multirow{3}{*}{$\mathbf{0}$} & $\mathrm{C}$ & $\mathrm{B}$ & $\mathrm{A}$ & $\mathrm{C}$ & $\mathrm{B}$ & A & $\mathrm{B}$ & A & A \\
\hline & $35.10 \mathrm{c}$ & $77.31 \mathrm{~d}$ & $89.94 \mathrm{~d}$ & $175.11 \mathrm{c}$ & $204.45 \mathrm{c}$ & $205.17 \mathrm{~d}$ & $210.20 \mathrm{~d}$ & $281.76 \mathrm{~d}$ & $295.11 \mathrm{~d}$ \\
\hline & $\mathrm{B}$ & A & A & A & A & A & $\mathrm{B}$ & $\mathrm{A}$ & A \\
\hline \multirow[t]{2}{*}{45} & $105.86 \mathrm{~b}$ & $136.95 \mathrm{c}$ & $141.66 \mathrm{c}$ & $256.13 \mathrm{~b}$ & $259.99 \mathrm{~b}$ & $247.81 \mathrm{c}$ & $361.99 \mathrm{c}$ & $396.93 \mathrm{c}$ & $389.47 \mathrm{c}$ \\
\hline & $\mathrm{C}$ & $\mathrm{B}$ & $\mathrm{A}$ & $\mathrm{B}$ & $\mathrm{B}$ & A & $\mathrm{C}$ & $\mathrm{B}$ & A \\
\hline \multirow[t]{2}{*}{90} & $134.87 \mathrm{a}$ & $156.59 \mathrm{~b}$ & $193.44 \mathrm{~b}$ & $266.12 \mathrm{~b}$ & $279.38 \mathrm{a}$ & $302.14 \mathrm{~b}$ & $400.99 \mathrm{~b}$ & $435.96 \mathrm{~b}$ & $495.59 \mathrm{~b}$ \\
\hline & $\mathrm{C}$ & $\mathrm{B}$ & A & $\mathrm{B}$ & $\mathrm{B}$ & A & $\mathrm{C}$ & $\mathrm{B}$ & A \\
\hline 135 & $140.96 \mathrm{a}$ & $183.86 \mathrm{a}$ & $255.30 \mathrm{a}$ & $282.87 \mathrm{a}$ & $297.46 \mathrm{a}$ & $339.16 \mathrm{a}$ & $423.82 \mathrm{a}$ & $481.32 \mathrm{a}$ & $594.45 \mathrm{a}$ \\
\hline
\end{tabular}

Table 7. Grain yield (ardab/fad.) and biological (ton /fad.) of the two maize hybrids as affected by nitrogen fertilizer levels, phosphorus fertilizer levels and their interactions in 2014, 2015 seasons and their combined data

\begin{tabular}{|c|c|c|c|c|c|c|}
\hline \multirow{2}{*}{$\begin{array}{l}\text { Main effects and } \\
\text { interactions }\end{array}$} & \multicolumn{3}{|c|}{ Grain yield (ardab /fad.) } & \multicolumn{3}{|c|}{ Biological (ton /fad.) } \\
\hline & 2014 & 2015 & Comb. & 2014 & 2015 & Comb. \\
\hline \multicolumn{7}{|l|}{ Maize hybrid (H) } \\
\hline SC 128 & $27.17 \mathrm{a}$ & $30.99 \mathrm{a}$ & $29.08 \mathrm{a}$ & $7.00 \mathrm{a}$ & $8.79 \mathrm{a}$ & $7.90 \mathrm{a}$ \\
\hline SC 176 & $25.48 \mathrm{~b}$ & $27.30 \mathrm{~b}$ & $26.39 \mathrm{~b}$ & $6.35 \mathrm{~b}$ & $6.00 \mathrm{~b}$ & $6.18 \mathrm{~b}$ \\
\hline F. test & $*$ & $* *$ & $* *$ & $* *$ & $* *$ & $* *$ \\
\hline \multicolumn{7}{|c|}{ Nitrogen level (kg N/fad.) $\mathbf{N}$} \\
\hline $\mathbf{0}$ & $16.99 \mathrm{~d}$ & $17.54 \mathrm{~d}$ & $17.27 \mathrm{~d}$ & $4.25 \mathrm{~d}$ & $4.39 \mathrm{~d}$ & $4.32 \mathrm{~d}$ \\
\hline 45 & $24.70 \mathrm{c}$ & $28.26 \mathrm{c}$ & $26.48 \mathrm{c}$ & $6.36 \mathrm{c}$ & $7.13 \mathrm{c}$ & $6.74 \mathrm{c}$ \\
\hline 90 & $30.30 \mathrm{~b}$ & $33.52 \mathrm{~b}$ & $31.91 \mathrm{~b}$ & $7.66 \mathrm{~b}$ & $8.43 \mathrm{~b}$ & $8.04 \mathrm{~b}$ \\
\hline 135 & $33.31 \mathrm{a}$ & $37.25 \mathrm{a}$ & $35.28 \mathrm{a}$ & $8.44 \mathrm{a}$ & $9.64 \mathrm{a}$ & $9.04 \mathrm{a}$ \\
\hline F. test & $* *$ & $* *$ & $* *$ & $* *$ & $* *$ & $* *$ \\
\hline \multicolumn{7}{|c|}{ Phosphorus level (kg. $\mathrm{P}_{2} \mathrm{O}_{5} /$ fad.) P } \\
\hline $\mathbf{0}$ & $21.42 \mathrm{c}$ & $23.58 \mathrm{c}$ & $22.50 \mathrm{c}$ & $5.69 \mathrm{c}$ & $6.20 \mathrm{c}$ & $5.94 \mathrm{c}$ \\
\hline 15.5 & $26.45 \mathrm{~b}$ & $29.52 \mathrm{~b}$ & $27.99 \mathrm{~b}$ & $6.71 \mathrm{~b}$ & $7.46 \mathrm{~b}$ & $7.08 \mathrm{~b}$ \\
\hline 31 & $31.11 \mathrm{a}$ & $34.33 \mathrm{a}$ & $32.72 \mathrm{a}$ & $7.64 \mathrm{a}$ & $8.53 \mathrm{a}$ & $8.09 \mathrm{a}$ \\
\hline F. test & $* *$ & $* *$ & $* *$ & $* *$ & $* *$ & $* *$ \\
\hline \multicolumn{7}{|l|}{ Interactions } \\
\hline $\mathbf{H} \times \mathbf{N}$ & NS & $* *$ & $* *$ & $*$ & $* *$ & $* *$ \\
\hline $\mathbf{H} \times \mathbf{P}$ & NS & $* *$ & $* *$ & NS & NS & NS \\
\hline $\mathbf{N} \times \mathbf{P}$ & $* *$ & $* *$ & $* *$ & $* *$ & $* *$ & $* *$ \\
\hline
\end{tabular}

*,** indicate significant at 0.05 and 0.01 levels of probability, respectively. $\mathrm{NS}=$ Not significant. 
meaning, the growth and yield parameters behavior found between the two hybrids could interpret much the high productivity of SC. 128 hybrid than SC. 176 one. These results are in conformity with those given by Golezani and Tajbakhsh (2012), Kandil (2013), and Modhej et al. (2014) as they recorded significant cultivar variations in final grain and biological yields/ unit area.

\section{Nitrogen level effect}

It is evident from Table 7 that each $\mathrm{N}$ increment up to $135 \mathrm{~kg} \mathrm{~N} /$ fad., produced a significant increase in both grain and biological yields/fad., in both seasons and their combined. According the combined analysis, adding 45, 90 and $135 \mathrm{~kg}$ $\mathrm{N} /$ fad., increased grain yield fad., by 53.33, 84.77 and $104.10 \%$ and biological yield by $65.01,86.11$ and $109.30 \%$, respectively. The response of grain and biological yields to the increase of $\mathrm{N}$ level reflected that observed in each of chlorophyll content, plant height, ear leaf area, leaf area index, ear length, stem diameter, row number /ear, grain number/row, hundred grain weight and grain and stover yields/plant as well as biological yield/plant Tables (1, 2, 3, 4, 5 and 6). Noval and Silem (2003) working on maize fertilization, stated that increasing $\mathrm{N}$ level up to $135 \mathrm{~kg} \mathrm{~N} / \mathrm{fad}$., decreased markedly number of days to $50 \%$ tasseling and silking if compared with both 45 and $90 \mathrm{~kg} \mathrm{~N}$ rates/fad., On the other hand, number of green leaves/plant showed marked increase by raising $\mathrm{N}$ levels/up to $135 \mathrm{~kg} \mathrm{~N} / \mathrm{fad}$., several authors reported significant increase in grain yield due to the increase of $\mathrm{N}$ up to $125 \mathrm{~kg}$ N/fad. El-Naggar et al. (2012) and El-Kholy (2015) also other got yield response due to adding 135 and $118 \mathrm{~kg} \mathrm{~N} /$ fad.

\section{Phosphors level effect}

It was previously mentioned that phosphorus fertilizer could control maize yield components. Increasing the $\mathrm{P}$ fertilizer rate reflected in a significant increase in grain and biological yields/fad., and this was true in both seasons and their combined. According to the combined data, adding 15.5 and $31 \mathrm{~kg} \mathrm{P}_{2} \mathrm{O}_{5} /$ fad., increased grain yield with abut 24.4 and $45.42 \%$ compared with unfertilized (control) since, grain yield was about 22.5, 27.99 and $32.72 \mathrm{ardab} / \mathrm{fad}$. Due to adding zero, 15.5 and $31 \mathrm{~kg} \quad \mathrm{P}_{2} \mathrm{O}_{5} /$ fad., respectively for biological yield, when the field was fertilized with 15.5 and $31 \mathrm{~kg} \mathrm{P}_{2} \mathrm{O}_{5} /$ fad., biological yield increased with about 19.19 and $36.19 \%$ compared to control, since, biological yield was about 5.99. 7.08 and $8.09 \%$ due to adding 15.5 and $31 \mathrm{~kg} \mathrm{P}_{2} \mathrm{O}_{5} /$ fad., respectively. Similar results were observed regarding chlorophyll content (Table 1), LAI and relative photosynthetic potential for grain yield/plant (Table 3), Plant height and ear leaf area (Table 2 ), ear length and stem diameter (Table 4), row number/ear and grain number/row as well as hundred grain weight (Table 5) and grain yield/ plant and biological yield/ plant. These results are in agreement with those reported by Khan $e t$ al. (2005), Ahmad et al. (2007), Hussein (2009) and Khan et al. (2014) as they reported significant increase in grain and biological yields/ fad., due to addition of $\mathrm{P}$ up to level of $\left(60,90,120 \mathrm{~kg} \mathrm{P}_{2} \mathrm{O}_{5} \mathrm{ha}^{-1}\right)$, respectively.

\section{Interaction effect}

In general, each $\mathrm{N}$ increment was effective to increase both grain and biological yields/fad., for the maize hybrids i.e. SC. 128 and SC. 176. It seemed that SC. 128 hybrid always had higher grain and biological yields/fad., than SC. 176 under the different levels, however the two maize hybrids had the same grain yield under check $\mathrm{N}$ treatment (Table 7-a).

It is evident from Table 7-b that any increase in phosphors fertilizer caused a significant increase in grain yield/fad., up to $31 \mathrm{~kg}_{2} \mathrm{O}_{5} /$ fad., for the two maize hybrids. In general SC. 128 hybrid always had higher grain yield/fad., than SC 176 under the different P levels (Table 6-b).

It is evident from Table 7-c that each $\mathrm{N}$ increment produced a significant increase in both grain and biological yields/fad., when maize plant fertilized by 15.5 or $31 \mathrm{~kg} \mathrm{P}_{2} \mathrm{O}_{5} /$ fad., whereas, no further significant increase could be obtained due to the increase of $\mathrm{N}$ level beyond $90 \mathrm{~kg} \mathrm{~N} /$ fad., at the check P treatment. Under all 
Table 7-a. Effect of interaction between maize hybrids and nitrogen fertilizer levels on grain yield (ardab/fad.) and biological (ton /fad.) (combined)

\begin{tabular}{lcccccccc}
\hline $\begin{array}{l}\text { Maize hybrid } \\
\text { H) }\end{array}$ & \multicolumn{9}{c}{ Grain yield (ardab /fad.) } & \multicolumn{7}{c}{ Biological yield (ton/fad.) } \\
\cline { 2 - 10 } & \multicolumn{1}{c}{$\mathbf{0}$} & $\mathbf{4 5}$ & $\mathbf{9 0}$ & $\mathbf{1 3 5}$ & $\mathbf{0}$ & $\mathbf{4 5}$ & $\mathbf{9 0}$ & $\mathbf{1 3 5}$ \\
\cline { 2 - 9 } & $\mathrm{D}$ & $\mathrm{C}$ & $\mathrm{B}$ & $\mathrm{A}$ & $\mathrm{D}$ & $\mathrm{C}$ & $\mathrm{B}$ & $\mathrm{A}$ \\
\hline \multirow{2}{*}{ SC 128 } & $17.58 \mathrm{a}$ & $28.56 \mathrm{a}$ & $33.55 \mathrm{a}$ & $36.64 \mathrm{a}$ & $4.84 \mathrm{a}$ & $7.60 \mathrm{a}$ & $8.96 \mathrm{a}$ & $10.19 \mathrm{a}$ \\
& $\mathrm{D}$ & $\mathrm{C}$ & $\mathrm{B}$ & $\mathrm{A}$ & $\mathrm{D}$ & $\mathrm{C}$ & $\mathrm{B}$ & $\mathrm{A}$ \\
SC 176 & $16.96 \mathrm{a}$ & $24.40 \mathrm{~b}$ & $30.27 \mathrm{~b}$ & $33.99 \mathrm{~b}$ & $3.81 \mathrm{~b}$ & $5.88 \mathrm{~b}$ & $7.13 \mathrm{~b}$ & $7.89 \mathrm{~b}$ \\
& & & & &
\end{tabular}

Table 7-b. Effect of interaction between maize hybrids and phosphorus fertilizer levels on grain yield (ardab /fad.) (combined)

\begin{tabular}{|c|c|c|c|}
\hline \multirow{3}{*}{$\begin{array}{l}\text { Maize hybrid } \\
\text { (H) }\end{array}$} & \multicolumn{3}{|c|}{ Grain yield (ardab/fad.) } \\
\hline & \multicolumn{3}{|c|}{$P$ level (kg $\mathrm{P}_{2} \mathrm{O}_{5} /$ fad.) } \\
\hline & $\mathbf{0}$ & $\mathbf{1 5 . 5}$ & 31 \\
\hline \multirow{3}{*}{ SC 128} & $\mathrm{C}$ & $\mathrm{B}$ & $\mathrm{A}$ \\
\hline & $23.81 \mathrm{a}$ & $28.88 \mathrm{a}$ & $34.56 \mathrm{a}$ \\
\hline & $\mathrm{C}$ & $\mathrm{B}$ & A \\
\hline SC 176 & $21.19 \mathrm{~b}$ & $27.09 \mathrm{~b}$ & $30.87 \mathrm{~b}$ \\
\hline
\end{tabular}

Table 7-c. Effect of interaction between nitrogen fertilizer levels and phosphorus fertilizer levels on grain yield (ardab/fad.) and biological (ton/fad.) (combined)

\begin{tabular}{|c|c|c|c|c|c|c|}
\hline \multirow{3}{*}{$\begin{array}{l}\text { N level } \\
\text { (kg N/fad.) }\end{array}$} & \multicolumn{3}{|c|}{ Grain yield (ardab /fad.) } & \multicolumn{3}{|c|}{ Biological yield (ton /fad.) } \\
\hline & \multicolumn{6}{|c|}{$P$ level (kg $\mathrm{P}_{2} \mathrm{O}_{5} /$ fad.) } \\
\hline & $\mathbf{0}$ & $\mathbf{1 5 . 5}$ & 31 & $\mathbf{0}$ & 15.5 & 31 \\
\hline & $\mathrm{C}$ & $\mathrm{B}$ & $\mathrm{A}$ & $\mathrm{C}$ & $\mathrm{B}$ & A \\
\hline \multirow[t]{2}{*}{$\mathbf{0}$} & $11.87 \mathrm{c}$ & $18.46 \mathrm{~d}$ & $21.47 \mathrm{~d}$ & $2.89 \mathrm{c}$ & $4.71 \mathrm{~d}$ & $5.38 \mathrm{~d}$ \\
\hline & $\mathrm{C}$ & $\mathrm{B}$ & A & $\mathrm{C}$ & $\mathrm{B}$ & A \\
\hline \multirow[t]{2}{*}{45} & $23.03 \mathrm{~b}$ & $26.80 \mathrm{c}$ & $29.61 \mathrm{c}$ & $6.13 \mathrm{~b}$ & $6.72 \mathrm{c}$ & $7.38 \mathrm{c}$ \\
\hline & $\mathrm{C}$ & $\mathrm{B}$ & $\mathrm{A}$ & $\mathrm{C}$ & $\mathrm{B}$ & $\mathrm{A}$ \\
\hline \multirow[t]{2}{*}{90} & $27.37 \mathrm{a}$ & $31.38 \mathrm{~b}$ & $36.98 \mathrm{~b}$ & $7.14 \mathrm{a}$ & $7.91 \mathrm{~b}$ & $9.08 \mathrm{~b}$ \\
\hline & $\mathrm{C}$ & $\mathrm{B}$ & $\mathrm{A}$ & $\mathrm{C}$ & $\mathrm{B}$ & $\mathrm{A}$ \\
\hline 135 & $27.75 \mathrm{a}$ & $35.30 \mathrm{a}$ & $42.81 \mathrm{a}$ & $7.61 \mathrm{a}$ & $9.01 \mathrm{a}$ & $10.51 \mathrm{a}$ \\
\hline
\end{tabular}


the four $\mathrm{N}$ levels, each $\mathrm{P}$ increment produced a significant increase in both grain and biological yields/fad.

\section{Ear Leaf Efficiency and Migration Coefficient}

The average effects of nitrogen and phosphorus levels on ear leaf efficiency and migration coefficient of the maize hybrids are shown in Table 8.

\section{Maize hybrid differences}

The two maize hybrids i.e. SC. 128 and SC. 176 had statistically the same ear leaf efficiency and migration coefficient in both seasons and their combined.

\section{Nitrogen level effect}

It is evident from Table 8 that each $\mathrm{N}$ increment up to $135 \mathrm{~kg} \mathrm{~N} /$ fad., produced a significant increase in both ear leaf efficiency and migration coefficient in both seasons and their combined. According to the combined analysis, adding 45, 90 and $135 \mathrm{~kg} \mathrm{~N} /$ fad., increased ear leaf efficiency by $50.00,64.28$ and $85.71 \%$ and migration coefficient by 30.77 , 4615 and $50.00 \%$, respectively. These result are in consistent with those reported on other yield attributes and yield component. Ogola et al. (2002), Ghanem et al. (2006) and Khan et al. (2014).

\section{Phosphors level effect}

Results in Table 8 show that increasing $\mathrm{P}$ level from zero to 15 and $31 \mathrm{~kg} \mathrm{P}_{2} \mathrm{O}_{5} /$ fad., caused significant increase in both ear leaf efficiency and migration coefficient. This was true in both seasons and their combined however, the second $\mathrm{P}$ increment failed to add a further significant increase in migration coefficient. Basing on the combined data, increasing $\mathrm{P}$ levels up to $31 \mathrm{~kg} \mathrm{P}_{2} \mathrm{O}_{5} / \mathrm{fad}$., increased ear leaf efficiency by 23.53 and $41.17 \%$ and migration coefficient by 24.13 and $31.03 \%$, respectively compared with the control. Similar findings were reported by Hussein (2009) and Zafar et al. (2011).

\section{Interaction effect}

It is evident from Table 8-a that growing under unfertilized with $\mathrm{N}$, ear leaf efficiency of SC. 176 was higher than that of SC. 128 hybrid, whereas, under the different levels of $\mathrm{N}$ both SC. 128 and SC. 176 had the same ear leaf efficiency and migration coefficient with the exception under $135 \mathrm{~kg} \mathrm{~N} / \mathrm{fad}$., where the latter had higher migration coefficient than the former hybrid. In this respect it is noticed that ear leaf efficiency value of both hybrids increased consistently by any increase in $\mathrm{N}$ fertilizer. This was always observed for migration coefficient in SC. 176 hybrid, but no further significant increase could be obtained due to the increase of $\mathrm{N}$ level beyond $45 \mathrm{~kg} \mathrm{~N} /$ fad., in maize hybrid SC. 128 (Table 8-b).

In general, any increase in phosphorus fertilizer caused a significant increase in ear leaf efficiency up to $31 \mathrm{~kg} \mathrm{P}_{2} \mathrm{O}_{5} /$ fad., for the two maize hybrids. SC. 176 hybrid had higher ear leaf efficiency than SC. 128 under $15.5 \mathrm{~kg}$ $\mathrm{P}_{2} \mathrm{O}_{5} /$ fad., but the two maize hybrids had the same ear leaf efficiency under the other $\mathrm{P}$ level i.e. check and $31 \mathrm{~kg} \mathrm{P}_{2} \mathrm{O}_{5} / \mathrm{fad}$.

In general, under growing maize with 15.5 and $31 \mathrm{~kg} \quad \mathrm{P}_{2} \mathrm{O}_{5} /$ fad., ear leaf efficiency responded to nitrogen fertilizer up to $135 \mathrm{~kg}$ $\mathrm{N} /$ fad., but only increased till $90 \mathrm{~kg} \mathrm{~N} / \mathrm{fad}$, under without $\mathrm{P}$ treatment. Under all the four $\mathrm{N}$ levels, each $\mathrm{P}$ increment produced a significant increase in the ear leaf efficiency.

Each $\mathrm{N}$ increment produced a significant increase in migration coefficient under the high level of phosphors $31 \mathrm{~kg} \mathrm{P}_{2} \mathrm{O}_{5} /$ fad., where, no farther significant increase could be obtained due to the increase of $\mathrm{N}$ level beyond $45 \mathrm{~kg}$ $\mathrm{N} /$ fad., under $15.5 \mathrm{~kg}_{2} \mathrm{O}_{5} /$ fad., treatment, but only increased till $90 \mathrm{~kg} \mathrm{~N} /$ fad., under without $P$ application. Also, each $\mathrm{P}$ increment produced a significant increase in migration coefficient when maize plant fertilized by $135 \mathrm{~kg} \mathrm{~N} /$ fad., whereas migration coefficient was affected by the first $\mathrm{P}$ increment under the other $\mathrm{N}$ treatment i.e. check 45 and $90 \mathrm{~kg} \mathrm{~N} /$ fad., (Table 8-c). 
Table 8. Ear leaf efficiency and migration coefficient of the two maize hybrids as affected by nitrogen, phosphorus fertilizer levels and their interactions in 2014, 2015 seasons and their combined data

\begin{tabular}{lcccccc}
\hline $\begin{array}{l}\text { Main effects and } \\
\text { interactions }\end{array}$ & \multicolumn{3}{c}{ Ear leaf efficiency } & \multicolumn{3}{c}{ Migration coefficient } \\
\cline { 2 - 8 } & $\mathbf{2 0 1 4}$ & $\mathbf{2 0 1 5}$ & Comb. & $\mathbf{2 0 1 4}$ & $\mathbf{2 0 1 5}$ & Comb. \\
\hline Maize hybrid (H) & & & & & & \\
$\quad$ SC 128 & 0.20 & 0.21 & 0.21 & 0.33 & 0.35 & 0.34 \\
$\quad$ SC 176 & 0.20 & 0.22 & 0.21 & 0.33 & 0.36 & 0.35 \\
F. test & NS & NS & NS & NS & NS & NS
\end{tabular}

Nitrogen level (kg N/fad.) $\mathrm{N}$

$\begin{array}{lcccccc}\mathbf{0} & 0.13 \mathrm{~d} & 0.14 \mathrm{~d} & 0.14 \mathrm{~d} & 0.25 \mathrm{~d} & 0.27 \mathrm{~d} & 0.26 \mathrm{~d} \\ 45 & 0.20 \mathrm{c} & 0.22 \mathrm{c} & 0.21 \mathrm{c} & 0.34 \mathrm{c} & 0.35 \mathrm{c} & 0.34 \mathrm{c} \\ \mathbf{9 0} & 0.22 \mathrm{~b} & 0.24 \mathrm{~b} & 0.23 \mathrm{~b} & 0.36 \mathrm{~b} & 0.39 \mathrm{~b} & 0.38 \mathrm{~b} \\ \mathbf{1 3 5} & 0.26 \mathrm{a} & 0.27 \mathrm{a} & 0.26 \mathrm{a} & 0.38 \mathrm{a} & 0.41 \mathrm{a} & 0.39 \mathrm{a} \\ \text { st } & * * & * * & * * & * * & * * & * *\end{array}$

Phosphorus level (kg. $\mathrm{P}_{2} \mathrm{O}_{5} /$ fad.) P

\begin{tabular}{lcccccc}
$\mathbf{0}$ & $0.16 \mathrm{c}$ & $0.18 \mathrm{c}$ & $0.17 \mathrm{c}$ & $0.29 \mathrm{c}$ & $0.30 \mathrm{c}$ & $0.29 \mathrm{c}$ \\
$\mathbf{1 5 . 5}$ & $0.20 \mathrm{~b}$ & $0.22 \mathrm{~b}$ & $0.21 \mathrm{~b}$ & $0.35 \mathrm{a}$ & $0.37 \mathrm{~b}$ & $0.36 \mathrm{~b}$ \\
$\mathbf{3 1}$ & $0.24 \mathrm{a}$ & $0.25 \mathrm{a}$ & $0.24 \mathrm{a}$ & $0.36 \mathrm{a}$ & $0.40 \mathrm{a}$ & $0.38 \mathrm{a}$ \\
$\mathbf{s t}$ & $* *$ & $* *$ & $* *$ & $* *$ & $* *$ & $* *$ \\
$\mathbf{r a c t i o n s}$ & & & & & & \\
$\mathbf{H} \times \mathbf{N}$ & $*$ & $\mathrm{NS}$ & $*$ & $* *$ & $\mathrm{NS}$ & $* *$ \\
$\mathbf{H} \times \mathbf{P}$ & $\mathrm{NS}$ & $\mathrm{NS}$ & $*$ & $* *$ & $\mathrm{NS}$ & $\mathrm{NS}$ \\
$\mathbf{N} \times \mathbf{P}$ & $* *$ & $* *$ & $* *$ & $* *$ & $* *$ & $* *$ \\
\hline
\end{tabular}

*,** indicate significant at 0.05 and 0.01 levels of probability, respectively. $\mathrm{NS}=$ Not significant.

Table 8-a. Effect of interaction between maize hybrids and nitrogen fertilizer levels on ear leaf efficiency and migration coefficient (combined)

\begin{tabular}{|c|c|c|c|c|c|c|c|c|}
\hline \multirow{3}{*}{$\begin{array}{l}\text { Maize hybrid } \\
\text { (H) }\end{array}$} & \multicolumn{4}{|c|}{ Ear leaf efficiency } & \multicolumn{4}{|c|}{ Migration coefficient } \\
\hline & \multicolumn{8}{|c|}{ N level (kg N/ fad.) } \\
\hline & $\mathbf{0}$ & 45 & 90 & 135 & $\mathbf{0}$ & 45 & 90 & 135 \\
\hline & $\mathrm{D}$ & $\mathrm{C}$ & $\mathrm{B}$ & $\mathrm{A}$ & $\mathrm{B}$ & $\mathrm{A}$ & $\mathrm{A}$ & $\mathrm{A}$ \\
\hline \multirow[t]{2}{*}{ SC 128} & $0.12 \mathrm{~b}$ & $0.21 \mathrm{a}$ & $0.23 \mathrm{a}$ & $0.27 \mathrm{a}$ & $0.25 \mathrm{a}$ & $0.35 \mathrm{a}$ & $0.37 \mathrm{a}$ & $0.38 \mathrm{~b}$ \\
\hline & $\mathrm{D}$ & $\mathrm{C}$ & $\mathrm{B}$ & A & $\mathrm{D}$ & $\mathrm{C}$ & $\mathrm{B}$ & A \\
\hline SC 176 & $0.15 \mathrm{a}$ & $0.21 \mathrm{a}$ & $0.23 \mathrm{a}$ & $0.26 \mathrm{a}$ & $0.27 \mathrm{a}$ & $0.34 \mathrm{a}$ & $0.38 \mathrm{a}$ & $0.41 \mathrm{a}$ \\
\hline
\end{tabular}


Zagazig J. Agric. Res., Vol. 44 No. (1) 2017

Table 8-b. Effect of interaction between maize hybrids and phosphorus fertilizer levels on ear leaf efficiency (combined)

\begin{tabular}{|c|c|c|c|}
\hline \multirow{3}{*}{$\begin{array}{l}\text { Maize hybrid } \\
\text { (H) }\end{array}$} & \multicolumn{3}{|c|}{ Ear leaf efficiency } \\
\hline & \multicolumn{3}{|c|}{ P level (kg $\mathrm{P}_{2} \mathrm{O}_{5} /$ fad.) } \\
\hline & $\mathbf{0}$ & $\mathbf{1 5 . 5}$ & 31 \\
\hline & $\mathrm{C}$ & $\mathrm{B}$ & $\mathrm{A}$ \\
\hline \multirow[t]{2}{*}{ SC 128} & $0.17 \mathrm{a}$ & $0.21 \mathrm{~b}$ & $0.24 \mathrm{a}$ \\
\hline & $\mathrm{C}$ & B & A \\
\hline SC 176 & $0.17 \mathrm{a}$ & $0.22 \mathrm{a}$ & $0.25 \mathrm{a}$ \\
\hline
\end{tabular}

Table 8-c. Effect of interaction between nitrogen fertilizer levels and phosphorus fertilizer levels on ear leaf efficiency and mgration coefficient (combined)

\begin{tabular}{|c|c|c|c|c|c|c|}
\hline \multirow{3}{*}{$\begin{array}{l}\text { N level } \\
\text { (kg N/fad.) }\end{array}$} & \multicolumn{3}{|c|}{ Ear leaf efficiency } & \multicolumn{3}{|c|}{ Migration coefficient } \\
\hline & \multicolumn{6}{|c|}{$P$ level (kg $\mathrm{P}_{2} \mathrm{O}_{5} /$ fad.) } \\
\hline & $\mathbf{0}$ & 15.5 & 31 & $\mathbf{0}$ & $\mathbf{1 5 . 5}$ & 31 \\
\hline & $\mathrm{C}$ & $\mathrm{B}$ & $\mathrm{A}$ & B & $\mathrm{A}$ & $\mathrm{A}$ \\
\hline \multirow[t]{2}{*}{$\mathbf{0}$} & $0.09 \mathrm{c}$ & $0.16 \mathrm{c}$ & $0.17 \mathrm{~d}$ & $0.18 \mathrm{c}$ & $0.29 \mathrm{~b}$ & $0.31 \mathrm{~d}$ \\
\hline & $\mathrm{C}$ & B & $\mathrm{A}$ & B & $\mathrm{A}$ & $\mathrm{A}$ \\
\hline \multirow[t]{2}{*}{45} & $0.18 \mathrm{~b}$ & $0.23 \mathrm{~b}$ & $0.22 \mathrm{c}$ & $0.30 \mathrm{~b}$ & $0.36 \mathrm{a}$ & $0.37 \mathrm{c}$ \\
\hline & $\mathrm{C}$ & B & $\mathrm{A}$ & B & $\mathrm{A}$ & A \\
\hline \multirow[t]{2}{*}{90} & $0.20 \mathrm{a}$ & $0.22 \mathrm{~b}$ & $0.26 \mathrm{~b}$ & $0.34 \mathrm{a}$ & $0.38 \mathrm{a}$ & $0.40 \mathrm{~b}$ \\
\hline & $\mathrm{C}$ & B & A & $\mathrm{C}$ & $\mathrm{B}$ & $\mathrm{A}$ \\
\hline 135 & $0.21 \mathrm{a}$ & $0.25 \mathrm{a}$ & $0.33 \mathrm{a}$ & $0.34 \mathrm{a}$ & $0.40 \mathrm{a}$ & $0.44 \mathrm{a}$ \\
\hline
\end{tabular}

\section{REFERENCES}

Abd-Alla, AA. (2005). Maize yield potentiality in response to bio and mineral nitrogen fertilizers under drip irrigation regimes in the newly reclaimed soils. J. Agric. Sci. Mansoura Univ., 30 (10): 5765-5779.

Abdou, E.M., A.A. Ibrahim, S.A.I. Ghanem, O.A.A. Zeiton and A.E.A. Omar (2012). Effect of planting density and nitrogen fertilization on yield and it's attributes of some yellow maize hybrids. Zagazig J. Agric. Res., 39 (6): 1033-1046.
Ahmad, M., N. Hussain, A. Jan, K. Ahmad and S. Hussain (2007). Response of maize to phosphorus levels and plant density. Sarhad J. Agric., 23 (1): 69 - 74.

Akmal, M., H.U. Rehman, F.M. Asim and H. Akbar (2010). Response of maize varieties to nitrogen application for leaf area profile, crop growth, yield and yield components. Pak. J. Bot., 42 (3): 1941-1947.

Amanullah, Z.M. and S.K. Khalil (2010). Timing and rate of phosphorus application influence maize phenology, yield and 
profitability in Northwest Pakistan. Int. J. Plant Prod., 4: 281-291.

Arif, M., M.T. Jan, N.U. Khan, H. Akbar, S.A. Khan, M.J. Khan, A. Khan, I. Munir, M. Saeed and A. Iqbal (2010). Impact of plant populations and nitrogen levels on maize. Pak. J. Bot., 42 (6): 3907-3913.

Atia, A.M. (2006). Effect of bio-chemical fertilization regimes on yield of maize. M.Sc. Thesis, Fac. Agric., Zagazig Univ., Egypt.

Attia, A.N.E., S.A. El-Mloursy, G.M.A. Mahgoub and M.M.B. Darwich (2009). Effect of ridge spacing and plant density for two maize hybrids. J. Agric. Sci. Mansoura Univ., 34 (7): 8073- 8080.

Azeez, J.O., M.T. Adetunji and S.T.O. Lagoke (2006). Response of low-nitrogen tolerant maize genotypes to nitrogen application in a tropical Alfisol in Northern Nigeria. Soil and Tillage Res., 91 (1): 181-185.

Badawi, M.A. and S.A. El-Moursy (1997). Nitrogen and phosphorus requirements for maize, Zea mays, L. grown in a newly reclaimed sandy soil. Mansoura Univ. J. Agric. Sci., 22: 659-671.

Bader, M.A. and S.A. Othman (2006): Effect of plant density, organic manure, bio and mineral nitrogen fertilizers on maize growth and yield and soil fertility. Ann. Agric. Sc., Moshtohor, Egypt, 44 (1): 75-88.

Bakht, J., S. Ahmad, M. Tariq, H. Akber and M. Shafi (2006). Response of maize to planting methods and $\mathrm{N}$ fertilizer. J. Agric. and Biol. Sci., 1 (3): Sept., 1-14.

Darwich, M.M.B. (2013). Effect of $\mathrm{N}$ rates, compost and humic acid treatments on growth and yield components of maize. Ph. D. Thesis in Agron., Fac. Agric. Mansoura Univ., Egypt.

Diab, M., M.I. Katta, M.S. Zeid and S.H.M. Abdel-Rassoul (1990). Corn response of the rate and placement of phosphate fertilization. J. Agric. Sci., Mansoura Univ., 15 : 21822187

Ding, L., K.J. Wang, G.M. Jiang, D.K. Biswas1, H. Xu, L.F. Li and Y.H. Li (2005). Effects of nitrogen deficiency on photosynthetic traits of maize hybrids released in different years. Ann. Bo., 96: 925-930.

El-Azab, A.A.S. (2012). Response of maize to organic and mineral fertilization under foliar application treatments. Ph. D. Thesis in Agron., Fac. Agric. Mansoura Univ., Egypt.

El-Far, H.A.R. (1996). Effect of some cultural practices on growth and yield of maize (Zea mays, L.). M.Sc. Thesis, Fac. Agric. Mansoura Univ., Egypt.

El-Kholy, A.S.M.M. (2015). Fertigated nitrogen level, organic manure, and plant population for drip irrigated maize in sandy soils. M.Sc. Thesis, Fac. Agric., Zagazig Univ., Egypt.

El-Moursy, R.S.A. (2013). Agronomic studies on maize. Ph. D. Thesis in Agron., Fac. Agric. Mansoura Univ., Egypt.

El-Murshedy, W.A. (2002). Response of some maize cultivars to nitrogen fertilization under two farming systems. J. Agric. Sci. Mansoura Univ., 27 (5): 2821-2835.

El-Nagar, G.R. (2002). Effect of nitrogen fertilizer and foliar application with micronutrients on white and yellow maize. Assiut J. Agric. Sci., 33(3) : 85- 102.

El-Naggar, N.Z.A., M.A. Mohamed, S.A. Mowafy and I.M. Abd El-Hameed (2012). Effect of FYM and $\mathrm{N}$ fertilizer on yield and land use efficiency of maize-soybean intercropping. J. Plant Prod., Mansoura Univ., 3 (5): 729 - 746.

El-Sheikh, F.T. (1998). Effect of soil application of nitrogen and foliar application with manganese on grain yield and quality of maize (Zea mays L.). Proc. $8^{\text {th }}$ Conf. Agron., Suez Canal Univ., Ismailia, Egypt, 28-29: Nov., 182-189.

El-Sobky, E.E.A.M. (2014). Effect of some agronomic practices on productivity of maize. Ph.D. Thesis, Fac. Agric., Zagazig Univ., Egypt.

FAOSTAT (2015). FAO Statistics Division, February 2015.

Freed, R.S.P., S.P. Eisensmith, S. Goetez, D. Reicosky, V.W. Smail and P. Wolberg. (1989). User's Guide to MSTAT-C A software program for the design, management and 
analysis of agronomic research experiments. Michigan State Univ., USA.

Ghanem, S.A.I., M.A. Gomaa, R.M. Aly and A.M. Atia (2006). Yield of maize in relation to photosynthate partitioning parameters. Zagazig J. Agric. Res., 33 (4): 645-663.

Ghimire, K.H., K.B. Koirala, S.B. Bk, H.K. Prasai and R.P. Poudel (2007). Full season maize varietal research in western hills of Nepal. Proceedings of the $25^{\text {th }}$ National Summer Crops Research Workshop on Maiz Research and Production in Nepal held at NARC, Khumaltar, Lalitpur, Nepal, June 2123, 147-156.

Golezani, K.G. and Z. Tajbakhsh (2012). Relationship of plant biomass and grain filling with grain yield of maize cultivars. Int. J. Agric. and Crop Sci., 4 (20): 15361539 .

Gomez, K.H. and A.A. Gomez (1984). Statistical Procedures for Agriculture Research. John Willy and Sons, Inc., New York.

Hegazy, M.H., S.A. Genaidy, A.A. Abd ElMagid and K.M. Khalil (1996). Nitrogen and phosphorus fertilization for corn (Zea mays L.) in relation to some untraditional fertilizer at Kafr El-Shikh soil. J. Agric. Sci. Mansoura Univ., 21: 3367-3372.

Hejazi, L. and A. Soleymani (2014). Effect of different amounts of nitrogen fertilizer on grain yield of forage corn cultivars in Isfahan. Int. J. Adv. Biol. and Biom. Res., 2 (3): 608-614.

Hussein, A.H.A. (2009). Phosphorus use efficiency by two varieties of corn at different phosphorus fertilizer application rates. Res. J. Appl. Sci., 4 (2): 85-93.

Ikramullah, M.I., A.A. Khakwani, M. Sadiq, I. Awan, M. Munir and A. Ghazanfarullah (2015). Effects of nitrogen fertilization rates on growth, quality and economic return of fodder maize (Zea mays L.). Sarhad J. Agric., 31 (1): 45-52.

Jackson, M.L. (1958). Soil Chemical Analysis. Prentice-Hall, Inc., England Cliffs, V.S. Library of Congress, USA.
Kandil, E.E.E. (2013). Response of some maize hybrids (Zea mays L.) to different levels of nitrogenous fertilization. J. Appl. Sci. Res., 9 (3): 1902-1908.

Khalil, M.A.G. (2001). Response of some yellow and white maize cultivars to plant densities and nitrogen fertilization. M.Sc. Thesis, Fac. Agric., Kafr El-Sheikh, Tanta Univ., Egypt.

Khan, A., F. Munsif, K. Akhtar, M.Z. Afridi, Z. Ahmad, S. Fahad, R. Ullah, F.K. Ahmed and M. Din (2014). Response of Fodder Maize to Various Levels of Nitrogen and Phosphorus. Ame. J. P. Sci., 5: 2323-2329.

Khan, M.A., M. Abid, N.A. Hussain and M.U. Masood (2005). Effect of phosphorous levels on growth and yield of maize (Zea mays L.) cultivars under saline conditions. Int. J. Agric. Biol., 7(3): 511-514.

Kogbe, J.O.S. and J.A. Adediran, (2003). Influence of nitrogen, phosphorus and potassium application on the yield of maize in the savanna zone of Nigeria. Afr. J. Biotechnol., 2 (10): 345-349.

Lindsay, W.L. and W.A. Norvell (1978). Development of DTPA soil test for micronutrients. Soil Sci. Soc. Ame. Proc., 42: $421-428$.

Mahgoub, G.M.A. and A.A. El-Shenawy (2006). Response of some maize hybrids to row spacing and plant density. Proc. of $1^{\text {st }}$ Conf. Field Crop Res. Inst., ARC, 22-24 Aug., Egypt, 285-293.

Masood, T., R. Gul, F. Munsif, F. Jalal, Z. Hussain and N. Noreen (2011). Effect of different phosphorus levels on the yield and yield components of maize. Sarhad J. Agric., 27: 167-180.

Matusso, J. and M. Materusse (2016). Growth and yield response of maize (Zea mays L.) to different nitrogen levels in acid soils. Acad. Res. J. Agric. Sci. and Res., 4 (2): 35-44.

Mckee, G.W. (1964). A coefficient for computing leaf area in hybrid corn. Agron. J., 56: 240-241.

Modhej, A., A. Kaihani and S. Lack (2014). Effect of nitrogen fertilizer on grain yield 
and nitrogen use efficiency in corn (Zea mays L.) hybrids under irrigated conditions. Proc. Natl. Acad. Sci., India, Sect. B Biol. Sci., 84 (3): 531-536.

Mohamed, N.A. (2004). Principle component and response curve analyses of some maize hybrids to different nitrogen fertilization levels and plant density. Bull. Fac. Agric., Cairo Univ., 55(4): 531-556.

Monje, O. A. and B. Bugbee (1992). Inherent imitations of nondestructive chlorophyll meters. A comparison of two types of meters. Hort. Sci., $27: 69-71$.

Mowafy, S.A.E. (2003). Response of some maize hyprids to nitrogen fertilizer splitting under drip irrigation system in sandy soils. Zagazig J. Agric. Res., 30 (1) : 17 - 34.

Mukhtar, T., M. Arif, S. Hussain, M. Tariq and K. Mehmood (2011). Effect of different rates of nitrogen and phosphorus fertilizers on growth and yield of maize. J. Agric. Res., 49 (3): 333-339.

Mukhtar, T., M. Arif, S. Hussain, M. Atif, S. Rehman and K. Hussain (2012). Yield and yield components of maize hybrids as influenced by plant spacing. J. Agric. Res., 50 (1): 59 - 69

Noval and Silem (2003). Growth and chemical properties of maize grains of some single crosses as affected by nitrogen and manure fertilization under sprinkler irrigation in a sandy soil. Egypt. J. Appl. Sci., 18 (5B): 583596.

Ogola, J.B.O., T.R. Wheeler and P.M. Harris (2002). Effects of nitrogen and irrigation on water use of maize crops. Field Crops Res., 78 (2): 105-117.

Olusegun, O.S. (2015). Nitrogen (N) and phosphorus (P) fertilizer application on maize (Zea mays L.) growth and yield at Ado-Ekiti, South-West, Nigeria. Ame. J. Exp. Agric., 6 (1): 22-29.

Oraby, F.T., M.F. Abd El-Maksoud and A.A. Sarhan (2005). Proper agronomic practices required to maximize productivity of some maize varieties in old and reclaimed soils: VResponse of ten maize hybrids to $\mathrm{N}$ fertilization under two locations. J. Prod. and Dev., 10 (1): 55-73.

Plenet, D., S. Etchebest, A. Mollier and S. Pellerin (2000). Growth analysis of maize field crops under phosphorus deficiency I. Leaf growth. Plant and Soil, 223: 117-130.

Popoola, O.P., K.K. Adesanya, T.M. Odusina and A.W Ayanrinde (2015). A quadratic regression analysis of the effect of three levels of NPK fertilizer on the yield of yellow maize. Ame. J. Comp. Mathematics, 5: 426-430.

Salem, M.A. (2000). Response of maize (Zea mays, L.) growth and yield to chemical and biofertilization. Zagazig J. Agric. Res., 24: 845-858.

Seadh, S.E., W.A.E. Abido and D.R. Abdulrahman (2015). The role of foliar application in reducing maize nitrogen requirements. J. Plant Prod., Mansoura Univ., 6 (7): 1168-1180.

Sharifi, R.S. and R. Taghizadeh (2009). Response of maize (Zea mays L.) cultivars to different levels of nitrogen fertilizer. J. Food, Agric. and Environ., 7 (3 and 4): 518 - 521.

Sharifi, R.S., M. Sedghi and A.O. Gholipouri (2009). Effect of population density on yield and yield attributes of maize hybrids. Res. J. Bio. Sci., 4 (4): 375 - 379.

Soliman, I.E. and H.S. Gharib (2011). Response of weeds and maize (Zea mays L.) to some weed control treatments under different nitrogen fertilizer rates. Zagazig J. Agric. Res., 38 (2): 249-271.

Sorkhi, F. and M. Fateh (2014). Effect of nitrogen fertilizer on yield components of maize. Int. J. Biosci., 5(6): 16-20.

Steel, R.G.D. and J.H. Torrie (1980). Principles and Procedures of Statistics: A Biometrical Approach. $2^{\text {nd }}$ Ed. McGraw-Hill Book Co New York USA.

Toaima, S.E.A. and S.A. Saleh (2003). Yield and yield components of maize and sunflower as affected by preceding crop and N- fertilizer levels. J. Agric. Sci. Mansoura Univ., 28 (4): 2467-2476. 
Vania, D., H. Kirchev, A. Sevov, A. Matev and N. Yardonova (2010). Genotypic response of maize hybrids to different nitrogen application under climatic conditions of Plovdiv Region. BALWOIS 2010 - Ohrid, Republic of Macedonia, 25(5) : $1-6$.

Waller, R.A. and D.B. Duncan (1969). A Bayes rule for symmetric multiple comparisons problems. J. Ame. Statist. Assoc., 64 14841503.

Watson, D.J. (1952). The physiological basis of variation in yield. Advan. Agron., $4: 101-$ 140.
Yosefi, K., M. Galavi, M. Ramrodi and S.R. Mousavi (2011). Effect of bio-phosphate and chemical phosphorus fertilizer accompanied with micronutrient foliar application on growth, yield and yield components of maize (Single Cross 704). Aust. J. of Crop Sci. Ajcs 5 (2): $175-180$.

Zafar, M., M.K. Abbasi and A. Zahid-urRehman (2011). Effect of combining organic materials with inorganic phosphorus sources on growth, yield, energy content and phosphorus uptake in maize at Rawalakot Azad Jammu and Kashmir, Pakistan. Arch. Appl. Sci. Res., 3 (2): 199-212.

\section{الاســتجـابة الفسـيولوجيـة لهجـن الـــرة الثـــاميـة للتسـميد النيتروجينــي والفـوسـفاتي

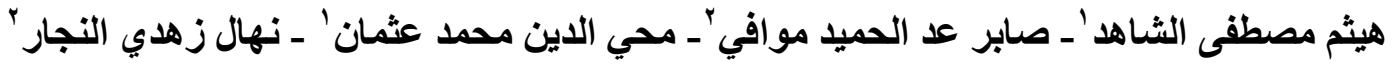

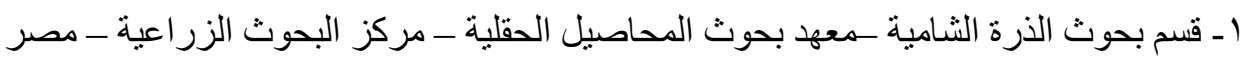 \\ بـ قسم المحاصيل - كلية الزراعة - جامعة الزقازيق - مصر}

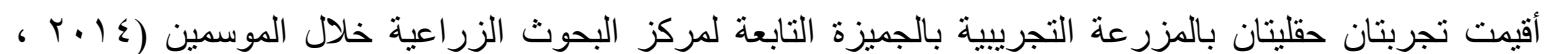

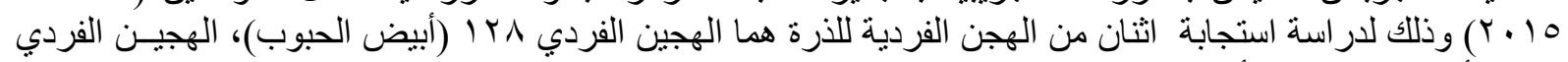

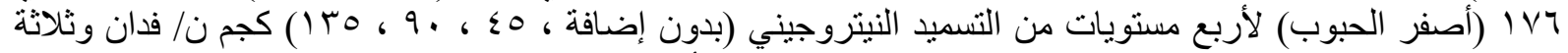

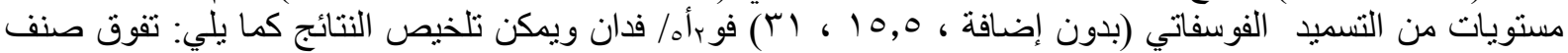

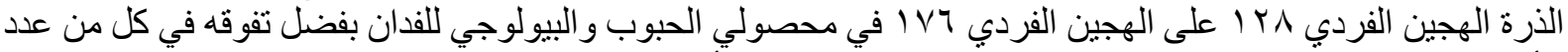

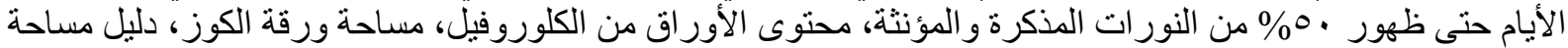

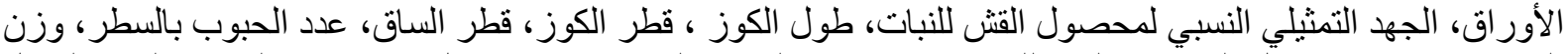

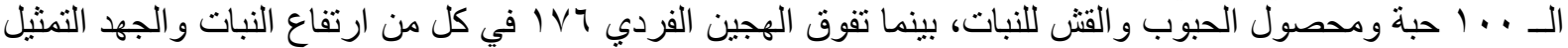

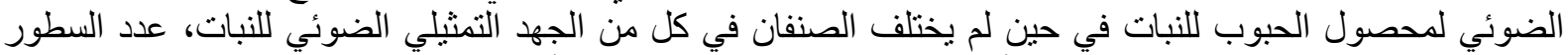

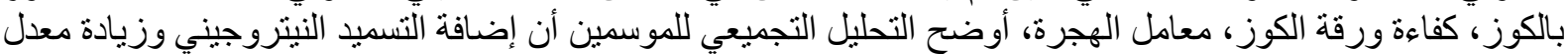

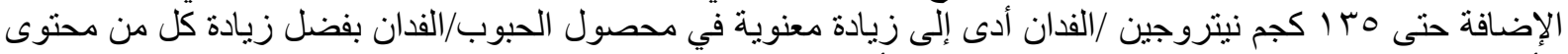

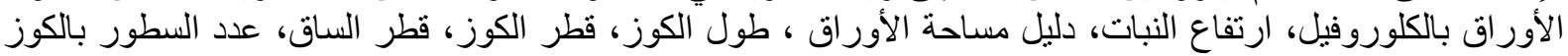

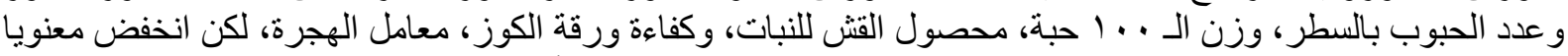

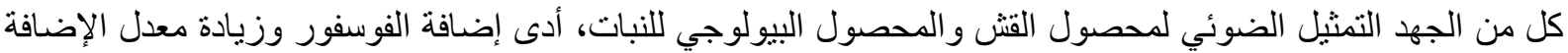

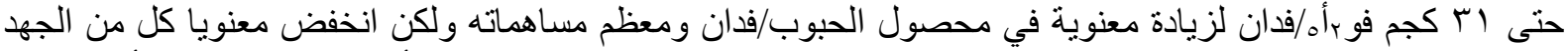

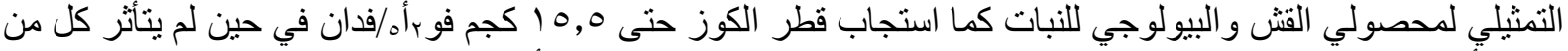

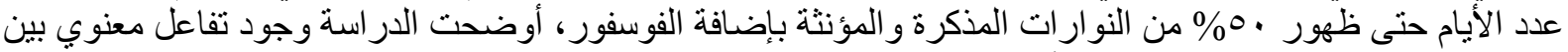

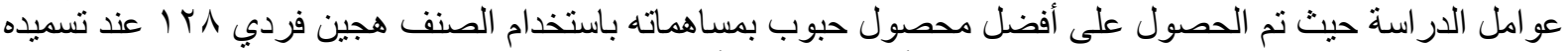

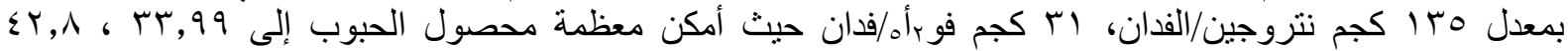

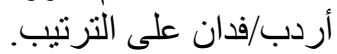

أستاذ المحاصبل - كلية الزر اعة - جامعسـة بنهـــا. أستاذ المحاصيل - كلية الزر اعة - - جامعة الزقازيق. 